\title{
Parental risk factors and anorectal malformations: systematic review and meta-analysis
}

\author{
Nadine Zwink, Ekkehart Jenetzky ${ }^{*}$ and Hermann Brenner
}

\begin{abstract}
Background: Anorectal malformations (ARM) are rare forms of congenital uro-rectal anomalies with largely unknown causes. Besides genetic factors, prenatal exposures of the parents to nicotine, alcohol, caffeine, illicit drugs, occupational hazards, overweight/obesity and diabetes mellitus are suspected as environmental risk factors.

Methods: Relevant studies published until August 2010 were identified through systematic search in PubMed, EMBASE, ISI Web of Knowledge and the Cochrane Library databases. Furthermore, related and cross-referencing publications were reviewed. Pooled odds ratios (95\% confidence intervals) were determined to quantify associations of maternal and paternal smoking, maternal alcohol consumption, underweight (body mass index [BMI] < 18.5), overweight (BMI 25-29.9), obesity (BMI $\geq 30$ ) and maternal diabetes mellitus with ARM using metaanalyses.
\end{abstract}

Results: 22 studies that reported on the association between prenatal environmental risk factors and infants born with ARM were included in this review. These were conducted in the United States of America $(n=12)$, Spain $(n=2)$, Sweden $(n=2)$, the Netherlands $(n=2)$, Japan $(n=1)$, France $(n=1)$, Germany $(n=1)$ and Hungary $(n=1)$. However, only few of these studies reported on the same risk factors. Studies were heterogeneous with respect to case numbers, control types and adjustment for covariates. Consistently increased risks were observed for paternal smoking and maternal overweight, obesity and diabetes, but not for maternal smoking and alcohol consumption. In meta-analyses, pooled odds ratios (95\% confidence intervals) for paternal smoking, maternal overweight, obesity, pre-gestational and gestational diabetes were 1.53 (1.04-2.26), 1.25 (1.07-1.47), 1.64 (1.35-2.00), 4.51 (2.55-7.97) and 1.81 (1.23-2.65), respectively.

Conclusion: Evidence on risk factors for ARM from epidemiological studies is still very limited. Nevertheless, the few available studies indicate paternal smoking and maternal overweight, obesity and diabetes to be associated with increased risks. Further, ideally large-scale multicentre and register-based studies are needed to clarify the role of key risk factors for the development of ARM.

Keywords: anorectal malformations, imperforate anus, anal atresia, birth defects, risk factors, pregnancy

\section{Introduction}

In recent years, a number of studies have shown that prenatal exposures of the parents are associated with an increased risk for having a malformed child. However, only few studies exist regarding the association with anorectal malformations (ARM).

ARM are rare birth defects concerning anus and rectum. Approximately 1 in 2,500 to 1 in 5,000 new born babies are affected [1-3]. Different degrees of severity

\footnotetext{
* Correspondence: e.jenetzky@dkfz.de

Division of Clinical Epidemiology and Aging Research, German Cancer Research Center, Heidelberg, Germany
}

are distinguished, ranging from mild anal stenosis over anal atresia with or without fistula to persistent cloaca or even cloacal exstrophy [4]. Furthermore, ARM frequently manifest with other malformations. Approximately $64 \%$ of all ARM patients are affected and have one or more additional extra-anal anomalies [5]. Previous studies have shown that associated malformations are more frequent in "high" defects that are complex and difficult to manage with a poor functional prognosis than in "low" defects that are less complex and easily treated with an excellent functional prognosis. Associated malformations mainly include the genitourinary 
system (21-61\% and more), spine and spinal cord (5-40\%), the rest of the gastrointestinal tract (10-25\%) and the heart (9-20\%) [6]. Anorectal malformations affect several socioeconomic and ethnic groups [7-11]. Boys seem to be at a slightly higher risk than girls (1.3:1) [12]. It is assumed that the defects occur during the 4th to 8th week of fetal development [13-18]. Current knowledge about the causes, however, is still sparse. In addition to genetic factors, prenatal exposures of the parents to tobacco, alcohol, caffeine, illicit drugs, overweight/obesity, diabetes mellitus and occupational hazards are subject to ongoing debate as potential environmental risk factors, in particular because the few existing studies are based on retrospectively collected data from individual centers only.

We conducted a systematic review and meta-analysis of epidemiological studies to summarize current evidence on the relationship between parental risk factors and anorectal malformations and to identify knowledge deficits that need to be addressed in future research.

\section{Methods}

\section{Identification of studies and study selection}

A literature search was carried out to identify epidemiological studies assessing the association between seven prenatal exposures of parents that have been suggested to be environmental risk factors for anorectal malformations: smoking, alcohol, caffeine, illicit drugs, overweight/obesity, diabetes and occupational hazards. Relevant studies published in English were systematically searched in PubMed, EMBASE, ISI Web of Knowledge and the Cochrane Library databases by using various combinations of the following terms: (congenital malformation(s), congenital abnormality, congenital abnormalities, birth defect(s), anorectal malformation(s), anorectal atresia, anal atresia, imperforate anus) AND (smok*, nicotine, tobacco, cigarette $^{*}$, alcohol ${ }^{*}$, drink ${ }^{*}$, caffeine ${ }^{*}$, coffee ${ }^{*}$, illicit drug(s), drug (s), overweight, obesity, adiposity, diabetes (mellitus), diabetes type 2 , diabetes type 1 , type 2 diabetes, type 1 diabetes, gestational diabetes, pre-gestational diabetes, preexisting diabetes, occupational hazard(s), occupational risk (s), professional risk(s), job hazard(s), parental occupation, maternal occupation, paternal occupation). Duplicate articles were deleted. Each title and abstract was checked for relevance. The full text was reviewed if the abstract indicated that the article reported an association between ARM and one of the previously mentioned risk factors. Furthermore, the identified articles were reviewed for related articles and cross-referring publications.

\section{Inclusion criteria}

Articles were included if they reported on associations of anorectal malformations with at least one of the previously mentioned environmental risk factors. When available, data of ARM infants with isolated anomalies (no additional major defects) were preferred to data of ARM infants with multiple defects. Articles were excluded if the reported number of ARM cases was less than two. ARM infants analysed only in a group with other anomalies like intestinal or tracheo-esophageal atresias were also excluded because of concern that associations of risk factors with these anomalies might be different from associations with ARM. Searches were restricted to English-language articles.

\section{Data extraction}

Two reviewers independently assessed the articles and extracted the following key information in a standardized manner: first author, year, country, study design, characteristics of the study population, period of data acquisition, assessed risk factor(s) for ARM and the respective measures of odds ratio or prevalence ratio (see below), as well as covariates adjusted for in the analysis. Initial disagreements on classifications of study characteristics were resolved by discussion within the team of authors.

Associations between parental exposures and ARM are presented by odds ratios (OR) and their 95\% confidence intervals (CI). Alternatively, reported prevalence ratios (PR) are shown. Unadjusted values were recalculated by the Review Manager Software, version 5.0.24 (The German Cochrane Centre, Freiburg, Germany) to validate the results. When measures of associations were not explicitly reported, they were derived from data provided in the articles.

\section{Meta-analyses}

Meta-analyses were performed for risk factors for which results were available from at least two studies. Heterogeneity was assessed by the $\chi^{2}$ and $\mathrm{I}^{2}$ statistics. When the number of studies is low or when sample sizes are small, the power of the $\chi^{2}$ test is low. The $\mathrm{I}^{2}$ measure describes the proportion of total variation in effect estimates across studies that is due to heterogeneity rather than sampling error [19]. Fixed and random effects models were calculated by the $\mathrm{R}^{\odot}$ software, version 2.11.1 (The R Foundation for Statistical Computing, Vienna) using standard meta-analysis methods. The fixed effects model was used to estimate the variance of the summary odds ratio when study heterogeneity was low $\left(\mathrm{I}^{2} \leq 25\right)$ and the random effects model when study heterogeneity was moderate to high $\left(\mathrm{I}^{2}>25\right)[20,21]$. Indication of publication bias was assessed by Begg and Mazumdar rank correlation test [22] and Egger's test [23] $(\mathrm{P}<0.1)$.

\section{Results}

\section{Literature search result}

In total, 9,623 articles were found (figure 1). After removal of 3,475 duplicates, 6,148 titles and abstracts 


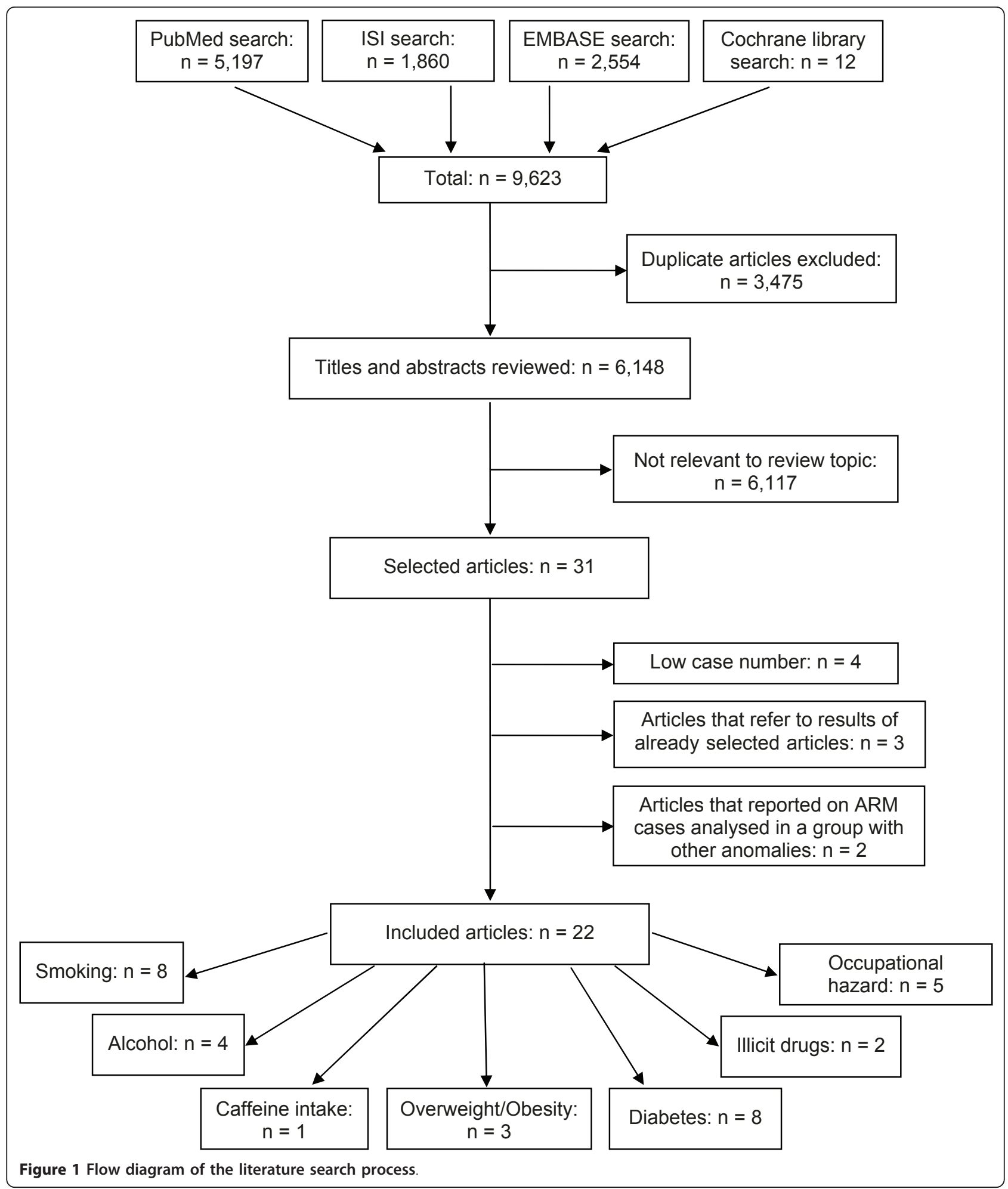

were reviewed. Thirty-one articles appeared to be potentially relevant for inclusion in the review. Of these, four articles were excluded because of too low case numbers $(\mathrm{n}<2)$, three articles because they referred to results of already selected articles and further two articles because they reported on ARM cases analysed in a group with other anomalies. Finally, 22 articles were included in the review. Among the included studies, eight provided data on the association of ARM with prenatal exposures to smoking, four to alcohol consumption, one to caffeine 
intake, two to illicit drug use, three to overweight/obesity, eight to diabetes mellitus and five to occupational hazards.

\section{Studies included in this review}

Details on the 22 studies, which were published from 1981 to June 2010, are shown in table 1. Studies were mainly conducted in the USA $(n=12)$. The remaining studies were conducted in Spain $(n=2)$, Sweden $(n=$ $2)$, the Netherlands $(n=2)$, Japan $(n=1)$, France $(n=$ $1)$, Germany $(n=1)$ and Hungary $(n=1)$. Recruitment was population-based in 16 studies and hospital-based in six studies. For data acquisition, nine studies relied on register-based data [1,7,24-30]. Data acquisition periods varied from one year [31] to 29 years [32].

Case numbers ranged from 14 ARM cases [33] to 564 ARM cases [31]. Children with known chromosomal anomalies were excluded in nine studies [26,27,34-40]. Twelve studies used healthy newborns or infants with no major birth defects as control group [7,25,28-30,34,35,37-41] and seven studies used malformed infants with other anomalies than ARM $[24,27,32,33,36,42,43]$. Controls of the remaining three studies were all infants born in the same settings during the respective study period $[1,26,31]$. Only six studies examined ARM infants with isolated anomalies [7,30,34,37,38,40].

\section{Findings for the reviewed risk factors}

Study results as well as the covariates adjusted for are shown in tables 2 to 8 .

\section{Cigarette consumption}

Seven studies reported on the association between maternal smoking before or during pregnancy and infants born with an anorectal malformation (table 2). Any smoking during pregnancy was significantly associated with ARM only in the study by Cornel et al. [27] (OR, 2.24; 95\% CI, 1.15-4.16; P = 0.01). In the study by Angerpointer et al. [42] different control groups were used. The comparison with one group of control infants with esophageal atresia, Hirschsprung's disease, omphalocele and gastroschisis showed a significant association between smoking at least five cigarettes per day and ARM (OR, 2.92; 95\% CI, 1.16-7.37). A similar trend was observed when using another control group of infants with esophageal atresia, stenosis/atresia of the small and large bowel, Hirschsprung's disease, omphalocele and gastroschisis (OR, 2.30; 95\% CI, 1.00-5.31). In contrast, no association at all was observed in analyses for any maternal smoking, regardless of the control group used. Honein et al. [31] observed a marginally increased risk for the maternal consumption of 6-10 cigarettes per day (PR, 1.38; 95\% CI, 1.00-1.90). The remaining studies could not confirm an association. Only two reviewed studies examined the association with paternal tobacco consumption. A significant association was observed by van Rooij et al. [39] (OR, 1.8; 95\% CI, 1.1-2.9; P = 0.01) whereas the study by Yuan et al. [30] could neither confirm this finding nor an association of ARM with smoking of both parents.

The result of the meta-analysis on the association between any maternal cigarette consumption and ARM infants is shown in figure 2. From the study by Angerpointer et al. [42] we used the OR calculated with the group of control infants with esophageal atresia, stenosis/atresia of the small and large bowel, Hirschsprung's disease, omphalocele and gastroschisis. The $\mathrm{I}^{2}$ statistic indicated heterogeneity across studies $\left(\chi^{2}=8.72 ; \mathrm{P}=\right.$ $\left.0.12 ; \mathrm{I}^{2}=42.6 \%\right)$. The estimated heterogeneity variance was $\operatorname{tau}^{2}=0.0284$. No significant association was observed in pooled analyses using the random effects model (OR, 1.03; 95\% CI, 0.83-1.29; P = 0.77). There was no evidence of publication bias (Kendall's tau = -0.56, P = 0.57; Egger's t value $=0.32, \mathrm{P}=0.77$ )

The result of the meta-analysis on the association between any paternal cigarette consumption and ARM infants is shown in figure 3 . The $\mathrm{I}^{2}$ statistic indicated low heterogeneity across the two studies $\left(\chi^{2}=1.21 ; \mathrm{P}=0.27\right.$; $\left.\mathrm{I}^{2}=17.5 \%\right)$. In meta-analysis, a weak association was found for any paternal cigarette consumption using a fixed effects model (OR, 1.53; 95\% CI, 1.04-2.26; $\mathrm{P}=0.03$ ).

\section{Alcohol consumption}

Among four studies assessing maternal alcohol consumption, the association with ARM was significant only in the one by Yuan et al. [30] (OR, 4.77; 95\% CI, 1.39-16.38) (table 3 ). The closer examination of alcohol quantity by Miller et al. [37] (no use, light use [ $\leq 1.5$ drinks per day], heavy use [ $>1.5$ drinks per day] and binge drinking $[\geq 5$ drinks on at least one occasion]) did not show any indication of a possible dose-response relationship.

The result of the meta-analysis on the association of any maternal alcohol consumption with ARM is shown in figure 4 . The $\mathrm{I}^{2}$ statistic indicated heterogeneity across studies $\left(\chi^{2}=6.74 ; \mathrm{P}=0.08 ; \mathrm{I}^{2}=55.5 \%\right)$. The estimated heterogeneity variance was $\operatorname{tau}^{2}=0.1172$. No significant association was observed in pooled analyses using the random effects model (OR, 1.17; 95\% CI, 0.71-1.91; $\mathrm{P}=0.55$ ). There was no evidence of publication bias (Kendall's tau = $1.36, \mathrm{P}=0.17$; Egger's t value $=1.37, \mathrm{P}=0.30$ ).

\section{Caffeine intake}

Only the study by Miller et al. [37] reported on a potential role of caffeine exposure (table 4). Although ARM was more common among children of mothers reporting on a periconceptional use of caffeine, the association was statistically significant for the intermediate exposure group 100-299 mg only (OR, 1.9; 95\% CI, 1.2-3.0). 


\begin{tabular}{|c|c|c|c|c|c|c|c|c|}
\hline \multirow[b]{3}{*}{ Ref. } & \multirow[b]{3}{*}{$\begin{array}{l}\text { First author, } \\
\text { year }\end{array}$} & \multicolumn{4}{|c|}{ Study population } & \multirow[b]{3}{*}{$\begin{array}{l}\text { Setting, control } \\
\text { type }\end{array}$} & \multirow[b]{3}{*}{ Data acquisition (period) } & \multirow[b]{3}{*}{ Assessed risk factor(s) } \\
\hline & & \multirow[b]{2}{*}{ Country } & \multicolumn{2}{|c|}{$\begin{array}{l}\text { No. } \\
\text { participants }\end{array}$} & \multirow[b]{2}{*}{ Age range } & & & \\
\hline & & & Cases & Controls & & & & \\
\hline [25] & Bánhidy, 2010 & Hungary & 231 & 38,151 & $<19->35$ & $\begin{array}{l}\text { population- } \\
\text { based, } \\
\text { no birth defects }\end{array}$ & $\begin{array}{l}\text { data from the Hungarian } \\
\text { Case-Control Surveillance } \\
\text { of Congenital Abnormalities } \\
\text { (1980-1996) }\end{array}$ & diabetes \\
\hline [26] & $\begin{array}{l}\text { Blomberg, } \\
2010^{*}\end{array}$ & Sweden & 401 & $1,049,181$ & $<20-\geq 45$ & $\begin{array}{l}\text { population- } \\
\text { based, } \\
\text { all infants }\end{array}$ & $\begin{array}{l}\text { data from the Swedish } \\
\text { Medical Birth Registries } \\
(1995-2007)\end{array}$ & overweight/obesity \\
\hline [35] & $\begin{array}{l}\text { Herdt-Losavio, } \\
2010\end{array}$ & USA & 328 & 3,833 & $<20-\geq 35$ & $\begin{array}{l}\text { multistate } \\
\text { population- } \\
\text { based, } \\
\text { no birth defects }\end{array}$ & $\begin{array}{l}\text { data from the National Birth } \\
\text { Defects } \\
\text { Prevention Study (NBDPS) } \\
\text { (1997-2003) }\end{array}$ & occupational hazard \\
\hline [39] & $\begin{array}{l}\text { van Rooij, } \\
2010\end{array}$ & Netherlands & 85 & 650 & $\geq 35$ & $\begin{array}{l}\text { hospital-based, } \\
\text { no major birth } \\
\text { defects }\end{array}$ & questionnaire $\left(1996-2008^{\ddagger}\right)$ & $\begin{array}{l}\text { smoking, alcohol, } \\
\text { overweight/obesity, } \\
\text { occupational hazard }\end{array}$ \\
\hline [37] & Miller, 2009 & USA & $\begin{array}{l}464, \\
216^{\#}\end{array}$ & 4,940 & $\leq 19-\geq 35$ & $\begin{array}{l}\text { multistate } \\
\text { population- } \\
\text { based, } \\
\text { no major birth } \\
\text { defects }\end{array}$ & $\begin{array}{l}\text { data from the National Birth } \\
\text { Defects } \\
\text { Prevention Study (NBDPS) } \\
(1997-2003)\end{array}$ & smoking, alcohol, caffeine \\
\hline [41] & $\begin{array}{l}\text { van Gelder, } \\
2009\end{array}$ & USA & $\begin{array}{l}456- \\
468\end{array}$ & 4,967 & $<20-\geq 35$ & $\begin{array}{l}\text { multistate } \\
\text { population- } \\
\text { based, } \\
\text { no major birth } \\
\text { defects }\end{array}$ & $\begin{array}{l}\text { data from the National Birth } \\
\text { Defects } \\
\text { Prevention Study (NBDPS), } \\
\text { collected by } \\
\text { telephone interview (1997- } \\
\text { 2003) }\end{array}$ & $\begin{array}{l}\text { illicit drugs of mothers } \\
\text { (between one month } \\
\text { before pregnancy } \\
\text { and the end of the third } \\
\text { month of pregnancy) }\end{array}$ \\
\hline [34] & Correa, 2008 & USA & $\begin{array}{l}230 \\
200^{\#}\end{array}$ & 4,689 & $<20-\geq 35$ & $\begin{array}{l}\text { multistate } \\
\text { population- } \\
\text { based, } \\
\text { no major birth } \\
\text { defects }\end{array}$ & $\begin{array}{l}\text { data from the National Birth } \\
\text { Defects } \\
\text { Prevention Study (NBDPS) } \\
\text { (1997-2003) }\end{array}$ & diabetes \\
\hline [1] & $\begin{array}{l}\text { Forrester, } \\
2007\end{array}$ & USA & 162 & 316,346 & N.A. & $\begin{array}{l}\text { state-wide } \\
\text { population- } \\
\text { based, } \\
\text { all live births }\end{array}$ & $\begin{array}{l}\text { data from the Hawaii Birth } \\
\text { Defects } \\
\text { Program (HBDP), collected } \\
\text { through review } \\
\text { of medical records (1986- } \\
\text { 2002) }\end{array}$ & $\begin{array}{l}\text { illicit drugs of mothers } \\
\text { (during } \\
\text { pregnancy and } 1 \text { year after } \\
\text { delivery) }\end{array}$ \\
\hline [32] & Frías, 2007 & USA & $\begin{array}{l}417^{\Delta} \\
427^{\prime \Delta}\end{array}$ & $\begin{array}{l}29,722^{\Delta} \\
30,509^{\Delta \Delta}\end{array}$ & N.A. & $\begin{array}{l}\text { hospital-based, } \\
\text { other malformed } \\
\text { infants }\end{array}$ & $\begin{array}{l}\text { data from the Spanish } \\
\text { Collaborative Study of } \\
\text { Congenital Malformations } \\
\text { (ECEMC) }(1976-2005)\end{array}$ & diabetes \\
\hline [40] & Waller, 2007 & USA & $\begin{array}{l}380 \\
77^{\#}\end{array}$ & 4,065 & $<18-\geq 35$ & $\begin{array}{l}\text { multistate } \\
\text { population- } \\
\text { based, } \\
\text { no birth defects }\end{array}$ & $\begin{array}{l}\text { data from the National Birth } \\
\text { Defects } \\
\text { Prevention Study (NBDPS) } \\
\text { (1997-2002) }\end{array}$ & overweight/obesity \\
\hline [7] & Correa, 2003 & USA & $\begin{array}{l}56, \\
32^{\#}\end{array}$ & 3,029 & $<20-\geq 30$ & $\begin{array}{l}\text { population- } \\
\text { based, } \\
\text { no birth defects }\end{array}$ & $\begin{array}{l}\text { data from the Metropolitan } \\
\text { Atlanta Congenital } \\
\text { Defects Program (MACDP) } \\
(1968-1980)\end{array}$ & diabetes \\
\hline [24] & Aberg, 2001 & Sweden & 15 & 600 & N.A. & $\begin{array}{l}\text { population- } \\
\text { based, } \\
\text { other malformed } \\
\text { infants }\end{array}$ & $\begin{array}{l}\text { data from the Swedish } \\
\text { Medical Birth Registries }{ }^{\dagger} \\
(1987-1997)\end{array}$ & diabetes \\
\hline [31] & Honein, 2001 & USA & 564 & $6,160,942$ & $<30-\geq 30$ & $\begin{array}{l}\text { population- } \\
\text { based, } \\
\text { all live births }\end{array}$ & $\begin{array}{l}\text { US public-use natality data } \\
\text { tapes } \\
\text { (National Vital Statistics } \\
\text { System, National } \\
\text { Centre for Health Statistics) } \\
\text { (1997-1998) }\end{array}$ & smoking \\
\hline
\end{tabular}


Table 1 Case-control and cross-sectional studies reporting on the association of ARM and environmental risk factors (Continued)

\begin{tabular}{|c|c|c|c|c|c|c|c|c|}
\hline$[36]$ & $\begin{array}{l}\text { Martínez-Frías, } \\
1998^{¥}\end{array}$ & Spain & 227 & 19,377 & N.A. & $\begin{array}{l}\text { hospital-based, } \\
\text { other malformed } \\
\text { infants }\end{array}$ & $\begin{array}{l}\text { data from the Spanish } \\
\text { Collaborative Study of } \\
\text { Congenital Malformations } \\
\text { (ECEMC) (1976-1995) }\end{array}$ & diabetes \\
\hline [38] & Stoll, 1997 & France & $\begin{array}{l}108 \\
51^{\#}\end{array}$ & 108 & $\begin{array}{l}\text { F: mean age } \\
26.9, \\
\text { M: mean age } \\
29.9\end{array}$ & $\begin{array}{l}\text { hospital-based, } \\
\text { no birth defects }\end{array}$ & interview (1979-1995) & $\begin{array}{l}\text { smoking, alcohol, diabetes, } \\
\text { X-ray examinations }\end{array}$ \\
\hline$[27]$ & Cornel, 1996 & Netherlands & 52 & 3,962 & $\leq 20-\geq 40$ & $\begin{array}{l}\text { population- } \\
\text { based, } \\
\text { other malformed } \\
\text { infants }\end{array}$ & $\begin{array}{l}\text { data from the Northern } \\
\text { Netherlands } \\
\text { (NNL) (1981-1994) }\end{array}$ & smoking \\
\hline$[29]$ & $\begin{array}{l}\text { Schnitzer, } \\
1995\end{array}$ & USA & 70 & 2,279 & $\begin{array}{l}F:<20-\geq 40 \\
M:<20-\geq 45\end{array}$ & $\begin{array}{l}\text { population- } \\
\text { based, } \\
\text { no birth defects }\end{array}$ & $\begin{array}{l}\text { data from the Metropolitan } \\
\text { Atlanta Congenital } \\
\text { Defects Program (MACDP) } \\
(1968-1980)\end{array}$ & occupational hazard \\
\hline$[30]$ & Yuan, 1995 & Japan & $\begin{array}{l}84 \\
49^{\#}\end{array}$ & 174 & $\begin{array}{l}F: 29.1 \pm 4.9 \\
M: 32.1 \pm 5.6\end{array}$ & $\begin{array}{l}\text { population- } \\
\text { based, } \\
\text { no birth defects }\end{array}$ & $\begin{array}{l}\text { data from the Kanangawa } \\
\text { Birth Defects } \\
\text { Monitoring Program (KAMP) } \\
(1989-1994)\end{array}$ & smoking, alcohol \\
\hline$[43]$ & $\begin{array}{l}\text { Martínez-Frías, } \\
1994^{*}\end{array}$ & Spain & 196 & 18,563 & N.A. & $\begin{array}{l}\text { hospital-based, } \\
\text { other malformed } \\
\text { infants }\end{array}$ & $\begin{array}{l}\text { data from the Spanish } \\
\text { Collaborative Study of } \\
\text { Congenital Malformations } \\
\text { (ECEMC) (1976-1992) }\end{array}$ & diabetes \\
\hline$[28]$ & Matte, 1993 & USA & 103 & 2,403 & $<20->35$ & $\begin{array}{l}\text { population- } \\
\text { based, } \\
\text { no birth defects }\end{array}$ & $\begin{array}{l}\text { data from the Metropolitan } \\
\text { Atlanta Congenital } \\
\text { Defects Program (MACDP) } \\
(1968-1980)\end{array}$ & occupational hazard \\
\hline [33] & Shiono, 1986 & USA & 14 & 578 & N.A. & $\begin{array}{l}\text { population- } \\
\text { based, } \\
\text { other malformed } \\
\text { infants }\end{array}$ & $\begin{array}{l}\text { data from the Kaiser- } \\
\text { Permanente } \\
\text { Birth Defects Study (1974- } \\
\text { 1977) }\end{array}$ & smoking \\
\hline$[42]$ & $\begin{array}{l}\text { Angerpointer, } \\
1981\end{array}$ & Germany & $\begin{array}{l}78 \\
78 \\
78 \\
78\end{array}$ & $\begin{array}{l}210^{*} \\
169^{* *} \\
75^{* * *} \\
53^{* * * *}\end{array}$ & $<20->40$ & $\begin{array}{l}\text { hospital-based, } \\
\text { other malformed } \\
\text { infants }\end{array}$ & questionnaire (1970-1974) & smoking \\
\hline
\end{tabular}

\# ARM infants with isolated (no additional major defects) anomaly.

† The Swedish Medical Birth Registry, the Swedish Register of Birth Defects (previously called the Registry of Congenital Malformations) and the National Patient Register (previously called the Hospital Discharge Registry).

\# Difference in case and control period: cases 1996-2008, controls 1996-2004.

$\triangle$ ARM infants for the examination of maternal pre-gestational diabetes.

$\triangle \triangle$ ARM infants for the examination of maternal gestational diabetes.

* Control group includes 41 infants with esophageal atresia, 41 with stenosis/atresia of the small and large bowel, 75 with Hirschsprung's disease, 28 with

omphalocele and 25 with gastroschisis.

** Control group includes 41 infants with esophageal atresia, 75 with Hirschsprung's disease, 28 with omphalocele and 25 with gastroschisis.

*** Control group includes 75 infants with Hirschsprung's disease.

**** Control group includes 28 infants with omphalocele and 25 with gastroschisis.

$¥$ Cross-sectional study.

$\mathrm{M}=$ male; $\mathrm{F}=$ female; $\mathrm{BMI}=$ body mass index; N.A. = not available.

\section{Illicit drugs}

Two studies reported on the association with maternal periconceptional illicit drug use (table 5). Results were inconsistent with tentatively reduced risks in the study by van Gelder et al. [41] and increased risks in the study by Forrester and Merz [1]. Due to the small sample size, confidence intervals were very wide in both studies. Nevertheless, significantly increased risks were found by Forrester and Merz [1] for marijuana use (OR, 10.57;
95\% CI, 2.87-38.96) as well as for cocaine use (OR, 6.01; 95\% CI, 1.05-34.27).

\section{Body weight}

Three studies reported on the association between maternal pre-pregnancy obesity $(B M I \geq 30)$ and ARM (table 6). Risks were consistently increased in two studies (Blomberg and Källén [26]: OR, 1.87; 95\% CI, 1.422.47; Waller et al. [40]: OR, 1.46; 95\% CI, 1.10-1.95). By 
Table 2 Associations between periconceptional exposures to tobacco

\begin{tabular}{|c|c|c|c|c|c|c|c|c|}
\hline \multirow[b]{2}{*}{ Ref. } & \multirow[b]{2}{*}{$\begin{array}{l}\text { First author, } \\
\text { year }\end{array}$} & \multirow[b]{2}{*}{ Exposure } & \multicolumn{2}{|l|}{ Maternal smoking } & \multicolumn{2}{|c|}{ Paternal smoking } & \multirow{2}{*}{$\begin{array}{l}\text { Smoking of both } \\
\text { parents } \\
\text { OR }[95 \% \mathrm{CI}]\end{array}$} & \multirow[b]{2}{*}{ Adjustment/matching factors } \\
\hline & & & OR $[95 \% \mathrm{Cl}]$ & $\begin{array}{l}P \\
\text { value }\end{array}$ & OR $[95 \% \mathrm{Cl}]$ & $\begin{array}{l}P \\
\text { value }\end{array}$ & & \\
\hline [39] & van Rooij, 2010 & $\begin{array}{l}\text { Cigarette consumption before or during } \\
\text { pregnancy }\end{array}$ & $0.8[0.5,1.3]$ & 0.61 & $1.8[1.1,2.9]^{\S}$ & 0.01 & - & - \\
\hline [37] & Miller, $2009^{\S \S}$ & $\begin{array}{l}\text { Non-smoker not exposed to ETS } \\
\text { Non-smoker exposed to ETS at home or } \\
\text { work } \\
\text { Non-smoker exposed to ETS at home } \\
\text { and work } \\
\text { Smoked }<0.5 \mathrm{pack} / \text { day } \\
\text { Smoked } \geq 0.5 \mathrm{pack} \text { day } \\
\text { Any smoking }\end{array}$ & $\begin{array}{l}1.0 \text { Reference } \\
1.1[0.8,1.5] \\
1.4[0.5,4.0] \\
1.0[0.5,1.8] \\
1.2[0.8,1.7] \\
1.1[0.8,1.6]\end{array}$ & - & - & & - & $\begin{array}{l}\text { None of the variables met the criteria } \\
\text { for confounding by the author; therefore, } \\
\text { only the unadjusted odds ratios were presented }\end{array}$ \\
\hline [31] & Honein, 2001 & $\begin{array}{l}\text { Any smoking } \\
1-5 \text { cigarettes/day } \\
6-10 \text { cigarettes/day } \\
11-20 \text { cigarettes/day } \\
\geq 21 \text { cigarettes/day }\end{array}$ & $\begin{array}{l}\text { PR: } 1.19[0.94,1.50] \\
\text { PR: } 0.95[0.60,1.50] \\
\text { PR: } 1.38[1.00,1.90] \\
\text { PR: } 1.19[0.80,1.79] \\
\text { PR: } 0.94[0.29,2.98]\end{array}$ & - & - & & - & $\begin{array}{l}\text { Adjusted for: maternal age, } \\
\text { education and race/ethnicity }\end{array}$ \\
\hline [38] & Stoll, 1997 & Any smoking & $0.98[0.94,1.02]$ & - & - & & - & - \\
\hline [27] & Cornel, 1996 & Any smoking & $2.24[1.15,4.16]$ & 0.01 & - & & - & - \\
\hline [30] & Yuan, 1995 & Any smoking & - & - & $1.14[0.59,2.18]$ & 0.70 & $1.75[0.63,4.87]$ & $\begin{array}{l}\text { Matched by: maternal age groups } \\
\text { (5-years interval), sex, parity } \\
\text { and season of birth }\end{array}$ \\
\hline [33] & Shiono, 1986 & Any smoking & $0.41[0.09,1.87]$ & 0.25 & - & & - & - \\
\hline [42] & $\begin{array}{l}\text { Angerpointer, } \\
1981\end{array}$ & $\geq 5$ cigarettes per day & $\begin{array}{l}0.95[0.45,1.99]^{*} \\
0.99[0.46,2.14]^{+} \\
1.20[0.47,3.10]^{+} \\
0.63[0.25,1.57]^{\#} \\
2.30[1.00,5.31]^{*} \\
2.92[1.16,7.37]^{+} \\
1.58[0.51,4.83]^{+}\end{array}$ & $\begin{array}{l}0.89 \\
0.98 \\
0.70 \\
0.32 \\
-\end{array}$ & - & & - & - \\
\hline
\end{tabular}

* Control group includes 41 infants with esophageal atresia, 41 with stenosis/atresia of the small and large bowel, 75 with Hirschsprung's disease, 28 with omphalocele and 25 with gastroschisis. † Control group includes 41 infants with esophageal atresia, 75 with Hirschsprung's disease, 28 with omphalocele and 25 with gastroschisis.

₹ Control group includes 75 infants with Hirschsprung's disease.

\# Control group includes 28 infants with omphalocele and 25 with gastroschisis.

$\S$ Paternal cigarette consumption 3 months before conception.

$\S \S$ Exposure during the month before pregnancy to the third month of pregnancy.

$\mathrm{ETS}=$ environmental tobacco smoke; $\mathrm{PR}=$ prevalence ratio. 
Table 3 Associations between periconceptional exposures to alcohol

\begin{tabular}{|c|c|c|c|c|c|c|c|c|}
\hline \multirow[b]{2}{*}{ Ref. } & \multirow[b]{2}{*}{$\begin{array}{l}\text { First } \\
\text { author, } \\
\text { year }\end{array}$} & \multicolumn{3}{|l|}{ Maternal alcohol use } & \multicolumn{3}{|l|}{$\begin{array}{l}\text { Paternal } \\
\text { alcohol use }\end{array}$} & \multirow[b]{2}{*}{$\begin{array}{l}\text { Adjustment/matching } \\
\text { factors }\end{array}$} \\
\hline & & Exposure & OR $[95 \% \mathrm{Cl}]$ & $\begin{array}{l}P \\
\text { value }\end{array}$ & Exposure & OR $[95 \% \mathrm{Cl}]$ & $\begin{array}{l}P \\
\text { value }\end{array}$ & \\
\hline [39] & $\begin{array}{l}\text { van Rooij, } \\
2010\end{array}$ & $\begin{array}{l}\text { Alcohol consumption before } \\
\text { or during pregnancy }\end{array}$ & $1.0[0.6,1.5]$ & 1.0 & $\begin{array}{l}\text { Alcohol } \\
\text { consumption } \\
\text { three } \\
\text { months before } \\
\text { conception }\end{array}$ & $1.3[0.7,2.5]$ & 0.33 & - \\
\hline [37] & $\begin{array}{l}\text { Miller, } \\
2009^{*}\end{array}$ & $\begin{array}{l}\text { Non-drinker } \\
\text { Average } \leq 1.5 \mathrm{drink} / \text { day } \\
\text { Average }>1.5 \mathrm{drink} / \text { day } \\
\geq 5 \text { alcoholic drinks } \\
\text { Drank any alcohol }\end{array}$ & $\begin{array}{l}\text { 1.0 Reference } \\
1.0[0.7,1.5] \\
1.2[0.9,1.6] \\
0.9[0.6,1.6] \\
0.9[0.7,1.2]\end{array}$ & - & - & - & - & $\begin{array}{l}\text { None of the variables met the } \\
\text { criteria } \\
\text { for confounding by the } \\
\text { author; therefore, } \\
\text { only the unadjusted odds } \\
\text { ratios were presented }\end{array}$ \\
\hline$[38]$ & Stoll, 1997 & Any alcohol & $1.25[0.07,21.04]$ & - & - & - & - & - \\
\hline$[30]$ & Yuan, 1995 & Any alcohol & $4.77[1.39,16.38]$ & - & - & - & - & $\begin{array}{l}\text { Matched by: maternal age } \\
\text { groups (5-years interval), } \\
\text { sex, parity and season of birth }\end{array}$ \\
\hline
\end{tabular}

* Exposure during the month before pregnancy to the third month of pregnancy.

categorizing maternal obesity into three classes (adipositas I [BMI 30-34.9], adipositas II [BMI 35-39.9] and morbid obesity [BMI $\geq 40]$ ), the closer examination by Blomberg and Källén [26] showed a particularly strong risk increase of ARM for morbid obesity (OR, 3.72; 95\% CI, 1.70-7.07). Among three studies assessing maternal overweight, a significant association with ARM was observed only in van Rooij et al. [39] (OR, 1.8; 95\% CI, 1.1-3.0). No such association was seen with paternal overweight in this study (OR, 0.8; 95\% CI, 0.5-1.3).

The result of the meta-analysis on the association of maternal underweight with ARM is shown in figure 5. The $\mathrm{I}^{2}$ statistic indicated low heterogeneity across the two studies $\left(\chi^{2}=1.01 ; \mathrm{P}=0.32 ; \mathrm{I}^{2}=0.5 \%\right)$. No significant association was observed in pooled analyses using the fixed effects model (OR, 0.96; 95\% CI, 0.65-1.43; P = 0.85).

The result of the meta-analysis on the association of maternal overweight with ARM is shown in figure 6 . The $\mathrm{I}^{2}$ statistic indicated low heterogeneity across the three studies $\left(\chi^{2}=2.25 ; \mathrm{P}=0.32 ; \mathrm{I}^{2}=11.3 \%\right)$. In meta-

Table 4 Associations between periconceptional exposures to caffeine intake

\begin{tabular}{|c|c|c|c|c|}
\hline \multirow[b]{2}{*}{ Ref. } & \multirow[b]{2}{*}{$\begin{array}{l}\text { First } \\
\text { author, } \\
\text { year }\end{array}$} & \multicolumn{2}{|c|}{$\begin{array}{l}\text { Maternal caffeine } \\
\text { exposure* }\end{array}$} & \multirow[b]{2}{*}{$\begin{array}{l}\text { Adjustment/matching } \\
\text { factors }\end{array}$} \\
\hline & & Exposure & OR $[95 \% \mathrm{Cl}]$ & \\
\hline$[37]$ & $\begin{array}{l}\text { Miller, } \\
2009\end{array}$ & $\begin{array}{l}<10 \mathrm{mg} \\
10-99 \mathrm{mg} \\
100-299 \\
\mathrm{mg} \\
\geq 300 \mathrm{mg}\end{array}$ & $\begin{array}{l}\text { 1.0 Reference } \\
1.4[0.9,2.3] \\
1.9[1.2,3.0] \\
1.5[0.9,2.7]\end{array}$ & $\begin{array}{l}\text { None of the variables } \\
\text { met the criteria for } \\
\text { confounding by the author; } \\
\text { therefore, only } \\
\text { the unadjusted odds ratios } \\
\text { were presented }\end{array}$ \\
\hline
\end{tabular}

* Caffeine intake per day in the year before pregnancy. analysis, a weak association was found for maternal overweight using a fixed effects model (OR, 1.25; 95\% CI, 1.07-1.47; P = 0.0054). There was no evidence of publication bias (Kendall's tau $=0.52, \mathrm{P}=0.60$; Egger's $\mathrm{t}$ value $=3.01, \mathrm{P}=0.20$ ).

The result of the meta-analysis on the association of maternal obesity with ARM is shown in figure 7 . The $I^{2}$ statistic indicated homogeneity across the three studies $\left(\chi^{2}=1.64 ; \mathrm{P}=0.44 ; \mathrm{I}^{2}=0 \%\right)$. In meta-analysis, a significant association was found for maternal obesity using a fixed effects model (OR, 1.64; 95\% CI, 1.35-2.00; $\mathrm{P}<$ $0.0001)$. There was no evidence of publication bias (Kendall's tau $=-0.52, \mathrm{P}=0.60$; Egger's $\mathrm{t}$ value $=-0.36$, $\mathrm{P}=0.78)$.

\section{Diabetes}

Among eight studies assessing maternal diabetes, six differentiated between pre-existing and gestational diabetes (table 7). The closer examination of results for pre-existing diabetes showed a significantly increased risk in the study by Frías et al. [32] (OR, 2.87; 95\% CI, 1.20-6.87; $\mathrm{P}=0.04)$. Correa et al. [34] and Aberg et al. [24] could confirm this finding (OR, 4.70; 95\% CI, 1.55-14.26 and OR, 8.18; 95\% CI, 3.86-17.34; $\mathrm{P}<0.00001)$ and also observed a significant association with gestational diabetes (OR, 1.91; 95\% CI, 1.02-3.56 and OR, 3.29; 95\% CI, 1.63-6.63; $\mathrm{P}=0.0008)$. Among four studies that reported on the association with any maternal diabetes, both studies by Correa et al. [7,34] found a significantly increased risk (OR, 2.15; 95\% CI, 1.31-3.55 and OR, 4.32; $95 \%$ CI, 1.50-12.47). Due to the small sample size, confidence intervals were very wide in all eight studies. 
Table 5 Associations between periconceptional exposures to illicit drugs

\begin{tabular}{|c|c|c|c|c|}
\hline \multirow[b]{2}{*}{ Ref. } & \multirow[b]{2}{*}{ First author, year } & \multicolumn{2}{|l|}{ Maternal illicit drug use } & \multirow[b]{2}{*}{ Adjustment/matching factors } \\
\hline & & Exposure & OR $[95 \% \mathrm{Cl}]$ & \\
\hline \multirow[t]{2}{*}[41]{} & van Gelder, 2009* & $\begin{array}{l}\text { Cannabis use } \\
\text { Cocaine use }\end{array}$ & $\begin{array}{l}0.7[0.4,1.2] \\
0.4[0.1,2.7]\end{array}$ & $\begin{array}{l}\text { Adjusted for: maternal age at delivery, race or ethnicity, } \\
\text { level of education, cigarette smoking, binge drinking, } \\
\text { pregnancy BMI and periconceptional folic acid use }\end{array}$ \\
\hline & & Stimulant use & $1.1[0.3,3.8]$ & $\begin{array}{l}\text { Adjusted for: maternal age at delivery, level of education, } \\
\text { binge drinking, pregnancy BMI and periconceptional } \\
\text { folic acid use }\end{array}$ \\
\hline$[1]$ & Forrester, $2007^{\S \dagger}$ & $\begin{array}{l}\text { Methamphetamine use } \\
\text { Cocaine use } \\
\text { Mariiuana use }\end{array}$ & $\begin{array}{l}3.19[0.87,11.73] \\
6.01[1.05,34.27] \\
10.57[2.87,38.96]\end{array}$ & - \\
\hline
\end{tabular}

* Exposures at any time between one month before pregnancy and the end of the third month of pregnancy (periconceptional period).

$\S$ Exposures during pregnancy and 1 year after delivery.

† Study reported on the ratio of the rate of illicit drug use among birth defect cases to the rate of illicit drug use among all deliveries. *We calculated the corresponding OR by data given in the article.

The result of the meta-analysis on the association of any maternal diabetes mellitus with ARM is shown in figure 8 . The $\mathrm{I}^{2}$ statistic indicated high heterogeneity across the four studies $\left(\chi^{2}=26.99 ; \mathrm{P}<0.0001 ; \mathrm{I}^{2}=\right.$ $88.9 \%)$. The estimated heterogeneity variance was $\operatorname{tau}^{2}=$ 1.0416. No significant association was observed in pooled analyses using the random effects model (OR, 1.01; 95\% CI, 0.33-3.12; $\mathrm{P}=0.99$ ). There was no evidence of publication bias (Kendall's tau $=0.0, \mathrm{P}=1.0$; Egger's t value $=-0.92, \mathrm{P}=0.45)$.

The result of the meta-analysis on the association of maternal pre-gestational diabetes with ARM is shown in figure 9 . The $\mathrm{I}^{2}$ statistic indicated moderate heterogeneity across the four studies $\left(\chi^{2}=4.13 ; \mathrm{P}=0.25 ; \mathrm{I}^{2}=\right.$ $27.4 \%)$. The estimated heterogeneity variance was $\operatorname{tau}^{2}=$ 0.0929. A strong association was observed in pooled analyses using the random effects model (OR, 4.51; 95\% CI, 2.55-7.97; $\mathrm{P}<0.0001)$. There was no evidence of publication bias (Kendall's tau $=-0.68, \mathrm{P}=0.50$; Egger's $\mathrm{t}$ value $=-1.15, \mathrm{P}=0.37$ ).

The result of the meta-analysis on the association of maternal gestational diabetes with ARM is shown in figure 10. The $\mathrm{I}^{2}$ statistic indicated moderate heterogeneity across the five studies $\left(\chi^{2}=5.71 ; \mathrm{P}=0.22 ; \mathrm{I}^{2}=30.0 \%\right)$. The estimated heterogeneity variance was tau ${ }^{2}=0.0570$. In meta-analysis, a significant association was found for maternal gestational diabetes using a random effects model (OR, 1.81; 95\% CI, 1.23-2.65; P = 0.0025). There was no evidence of publication bias (Kendall's tau $=0.98$, $\mathrm{P}=0.33$; Egger's t value $=0.85, \mathrm{P}=0.46$ ).

\section{Occupational hazard}

Five studies reported on a potential role of maternal and paternal occupational hazards (table 8). Herdt-Losavio et al. [35] found a significantly increased risk with maternal janitors and cleaners (OR, 1.82; 95\% CI, 1.06-3.10) and maternal scientists (OR, 2.38; 95\% CI, 1.24-4.55) and Schnitzer et al. [29] with paternal vehicle manufacturers (OR, 5.1; 95\% CI, 1.3-19.2). Van Rooij et al. [39] showed a suggestive association with ARM for maternal contact with industrial cleaning agents and solvents during pregnancy (OR, 2.9; 95\% CI, 0.9-9.3) and for paternal contact with exhaust fumes three months before conception (OR, 1.9; 95\% CI, 1.0-3.6). A significant inverse

Table 6 Associations between periconceptional exposures to body weight

\begin{tabular}{|c|c|c|c|c|}
\hline \multirow[b]{2}{*}{ Ref. } & \multirow[b]{2}{*}{ First author, year } & \multicolumn{2}{|c|}{ Maternal overweight/obesity } & \multirow[b]{2}{*}{ Adjustment/matching factors } \\
\hline & & Exposure & OR $[95 \% \mathrm{Cl}]$ & \\
\hline \multirow[t]{2}{*}[26]{} & Blomberg, 2010 & $\begin{array}{l}\text { BMI }<18.5 \\
\text { BMI } 18.5-24.9 \\
\text { BMI } 25-29.9 \\
\text { BMI } \geq 30\end{array}$ & $\begin{array}{l}1.22[0.63,2.13] \\
1.00 \text { Reference } \\
1.21[0.98,1.51] \\
1.87[1.42,2.47]\end{array}$ & $\begin{array}{l}\text { Adjusted for: maternal age, parity, smoking in early pregnancy } \\
\text { and year of birth using the Mantel-Haenszel } \\
\text { method }\end{array}$ \\
\hline & & $\begin{array}{l}\text { BMI } 30-34.9 \\
\text { BMI } 35-39.9 \\
\text { BMI } \geq 40\end{array}$ & $\begin{array}{l}1.77[1.29,2.44] \\
1.48[0.74,2.64] \\
3.72[1.70,7.07]\end{array}$ & Adjusted for: see above \\
\hline [39] & van Rooij, 2010** & $\begin{array}{l}\text { BMI } 25-29.9 \\
\text { BMI } \geq 30\end{array}$ & $\begin{array}{l}1.8[1.1,3.0] \\
1.4[0.6,3.2]\end{array}$ & $N / A^{*}$ \\
\hline$[40]$ & Waller, 2007 & $\begin{array}{l}\mathrm{BMI}<18.5 \\
\mathrm{BMI} 25-29.9 \\
\mathrm{BMI} \geq 30\end{array}$ & $\begin{array}{l}0.81[0.48,1.36] \\
1.19[0.92,1.55] \\
1.46[1.10,1.95]\end{array}$ & $\begin{array}{l}\text { Adjusted for: maternal age, ethnicity, education, parity, } \\
\text { smoking in the month prior to conception and supplemental } \\
\text { folic acid intake in the month prior to conception }\end{array}$ \\
\hline
\end{tabular}

* The association was not confounded by any covariable.

** More detailed information on the results was obtained directly through the author. 
Table 7 Associations between periconceptional exposures to diabetes mellitus

\begin{tabular}{|c|c|c|c|c|c|}
\hline \multirow[b]{2}{*}{ Ref. } & \multirow[b]{2}{*}{ First author, year } & \multicolumn{3}{|c|}{ Maternal diabetes mellitus } & \multirow[b]{2}{*}{ Adjustment/matching factors } \\
\hline & & Exposure & OR $[95 \% \mathrm{Cl}]$ & $P$ value & \\
\hline$[25]$ & Bánhidy, 2010 & Gestational diabetes & $2.2[0.7,6.8]$ & - & $\begin{array}{l}\text { Adjusted for: maternal age and employment status, birth } \\
\text { order and maternal hypertension }\end{array}$ \\
\hline$[34]$ & Correa, 2008 & $\begin{array}{l}\text { Diabetes mellitus** } \\
\text { Pre-gestational diabetes } \\
\text { Gestational diabetes }\end{array}$ & $\begin{array}{l}2.15[1.31,3.55] \\
4.70[1.55,14.26] \\
1.91[1.02,3.56]\end{array}$ & 0.005 & $\begin{array}{l}\text { Adjusted for: maternal age, race/ethnicity, entry into prenatal } \\
\text { care, BMl, study center and household income }\end{array}$ \\
\hline$[32]$ & Frías, 2007* & $\begin{array}{l}\text { Diabetes mellitus** } \\
\text { Pre-gestational diabetes } \\
\text { Gestational diabetes }\end{array}$ & $\begin{array}{l}1.43[0.92,2.25] \\
2.87[1.20,6.87] \\
1.18[0.71,1.98]\end{array}$ & $\begin{array}{l}0.13 \\
0.04 \\
0.48\end{array}$ & - \\
\hline$[24]$ & Aberg, 2001 & $\begin{array}{l}\text { Pre-gestational diabetes } \\
\text { Gestational diabetes }\end{array}$ & $\begin{array}{l}8.18[3.86,17.34] \\
3.29[1.63,6.63]\end{array}$ & $\begin{array}{l}<0.00001 \\
0.0008\end{array}$ & - \\
\hline$[7]$ & Correa, 2003 & Diabetes mellitus & $4.32[1.50,12.47]$ & - & $\begin{array}{l}\text { Adjusted for: infant's period of birth, maternal race, age, } \\
\text { education, prenatal cigarette smoking and prenatal alcohol } \\
\text { consumption }\end{array}$ \\
\hline$[36]$ & Martínez-Frías, 1998 & Gestational diabetes & $\begin{array}{l}1.51[0.60,3.55] \\
1.56[0.49,4.40]^{\dagger} \\
1.27[0.20,5.54]^{\ddagger} \\
1.70[0.67,3.98]^{\#} \\
4.19[0.66,26.74]^{\S}\end{array}$ & - & - \\
\hline [38] & Stoll, 1997 & Diabetes mellitus & $0.01[0.02,1.38]$ & - & - \\
\hline [43] & Martínez-Frías, 1994* & Pre-gestational diabetes & $2.57[0.69,9.60]$ & 0.19 & - \\
\hline
\end{tabular}

* Studies reported on the quotient of congenital anomaly frequencies only (frequency ratio: FR). We calculated the corresponding OR by data given in the articles.

** Studies reported on pre-gestational and gestational diabetes. We calculated the OR for any diabetes mellitus by data given in the articles.

† Restricted to: maternal age ( $\leq 34$ years).

‡ Restricted to: maternal age ( $\geq 35$ years).

\# Restricted to: non-consanguineous parents.

$\S$ Restricted to: insulin treatment.

association with maternal exposure to X-ray examinations was reported in the study by Stoll et al. [38] (OR, 0.19; 95\% CI, 0.09-0.38). Due to the small sample size, confidence intervals were very wide in the studies by Herdt-Losavio et al. [35], Matte et al. [28], Schnitzer et al. [29] and van Rooij et al. [39].

\section{Discussion}

This systematic review and meta-analysis summarized the results of 22 studies on the association between prenatal environmental risk factors and infants born with an anorectal malformation reported between 1981 and June 2010. The majority of the studies were conducted in the United States. Case numbers ranged from 14 ARM cases in the study by Shiono et al. [33] to 564 ARM cases in the study by Honein et al. [31]. Studies were also heterogeneous with respect to control selection and adjustment for covariates. Meta-analysis was done for risk factors reported on in at least two studies, i.e. maternal and paternal smoking, maternal alcohol consumption, underweight, overweight, obesity, any maternal diabetes mellitus, pre-gestational and gestational diabetes. Consistently increased risks were observed for paternal smoking, maternal overweight, obesity and diabetes, but not for maternal smoking and alcohol consumption.
There is a great discrepancy in the reported results on the associations between maternal illicit drug use and ARM which impede comparability. Closer examination of the studies suggests that different data collection could lead to these different results. In the study by van Gelder et al. [41], mothers were interviewed by telephone by trained interviewers using a standardized questionnaire. Forrester and Merz [1] used registerbased data from the Hawaii Birth Defects Program where trained staff collected information on cases through review of medical records. Thus, only consumption that had to be severe enough to be recorded in routine medical records was ascertained. It appears conceivable that illicit drug use might have been asked for and recorded more often among mothers of children with malformations than among other mothers. The discrepancy found on the associations between maternal exposures to X-rays may likewise be partly resulting from different exposure definitions. Van Rooij et al. [39] examined the maternal occupational hazard to $\mathrm{X}$-rays during pregnancy whereas Stoll et al. [38] assessed women's own X-ray examinations during pregnancy.

When available, data on ARM infants with isolated anomalies (no additional major defects) were preferred in this review to data on ARM infants with multiple defects. Only six of the 22 reviewed studies looked at 
Table 8 Associations between periconceptional exposures to occupational hazard

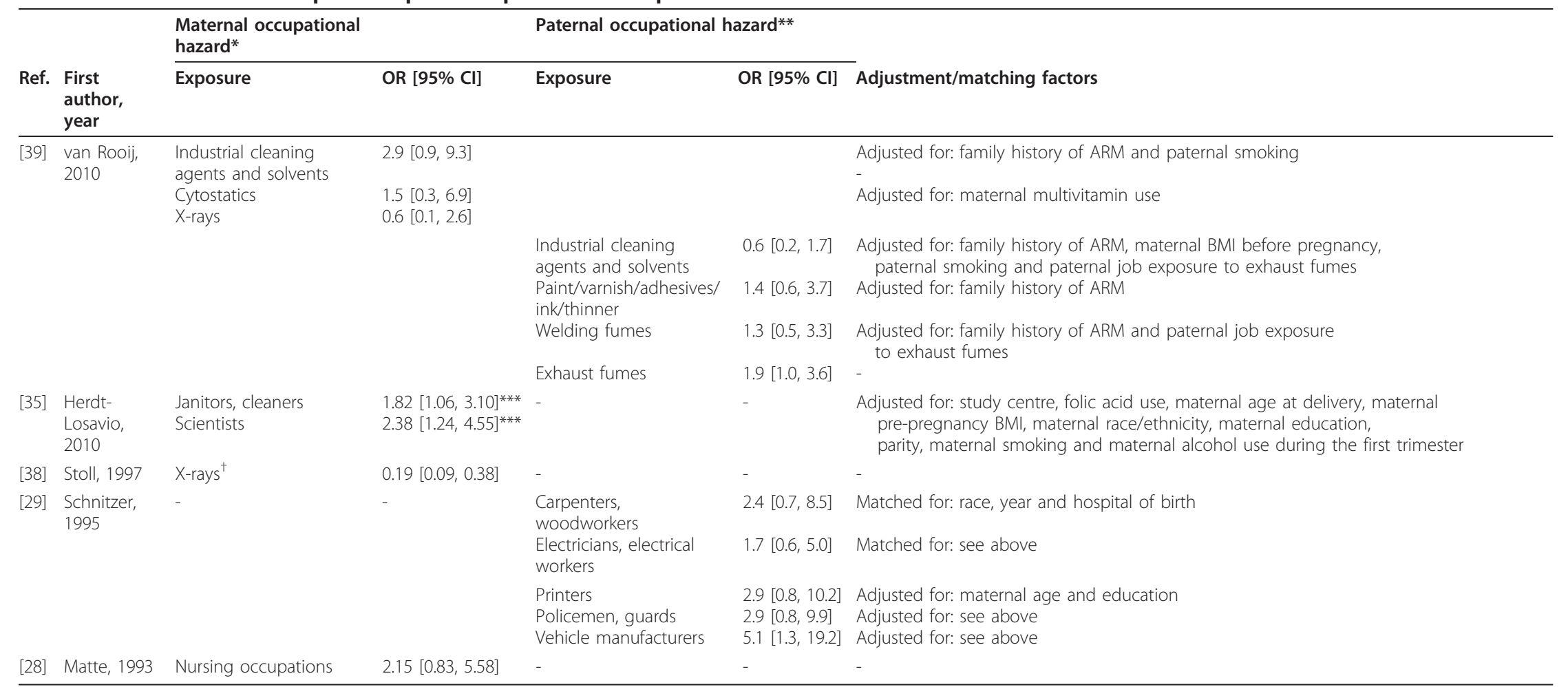

* Job exposure during pregnancy.

** Job exposure 3 months before conception.

*** Job exposure 1 month prior to conception through the end of the third month of pregnancy.

† Exposure to X-ray examinations. 


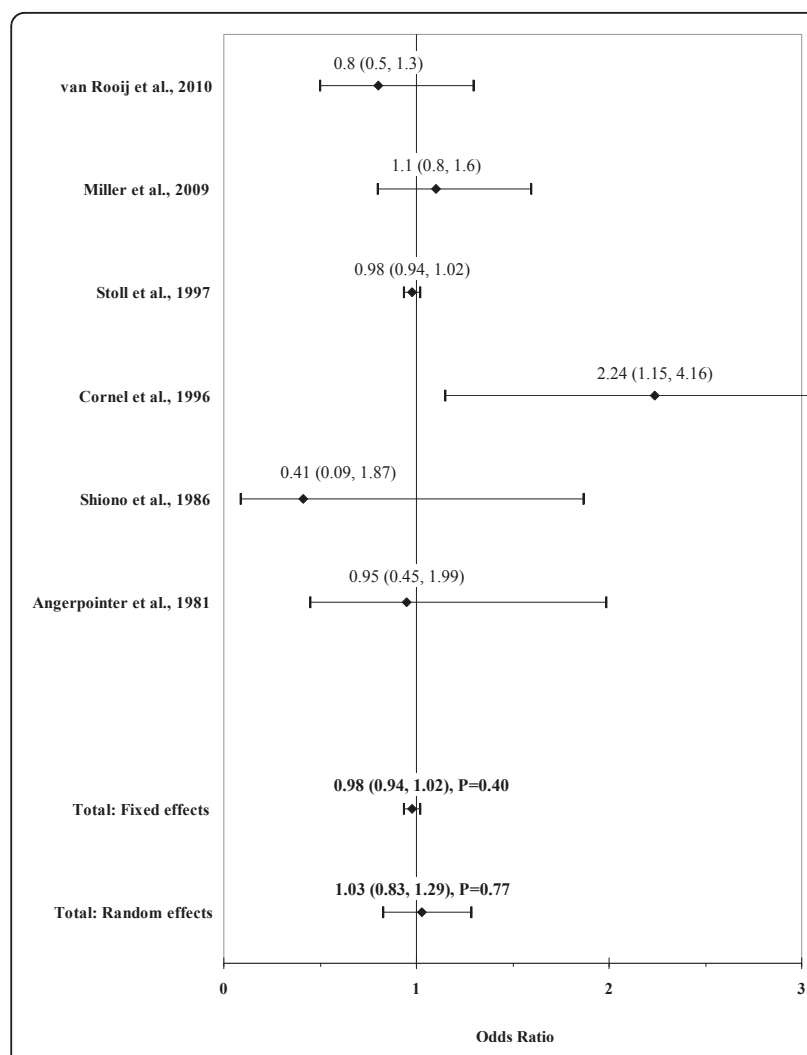

Figure 2 Forest plot for maternal cigarette consumption.

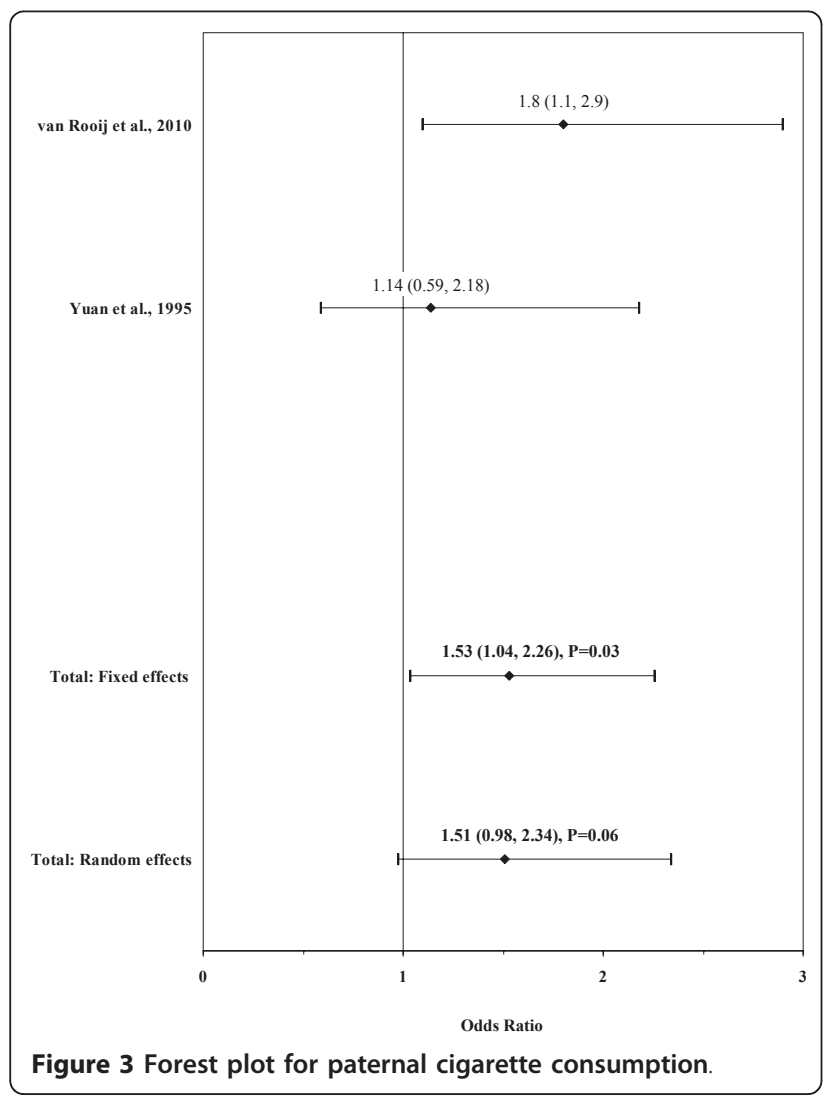

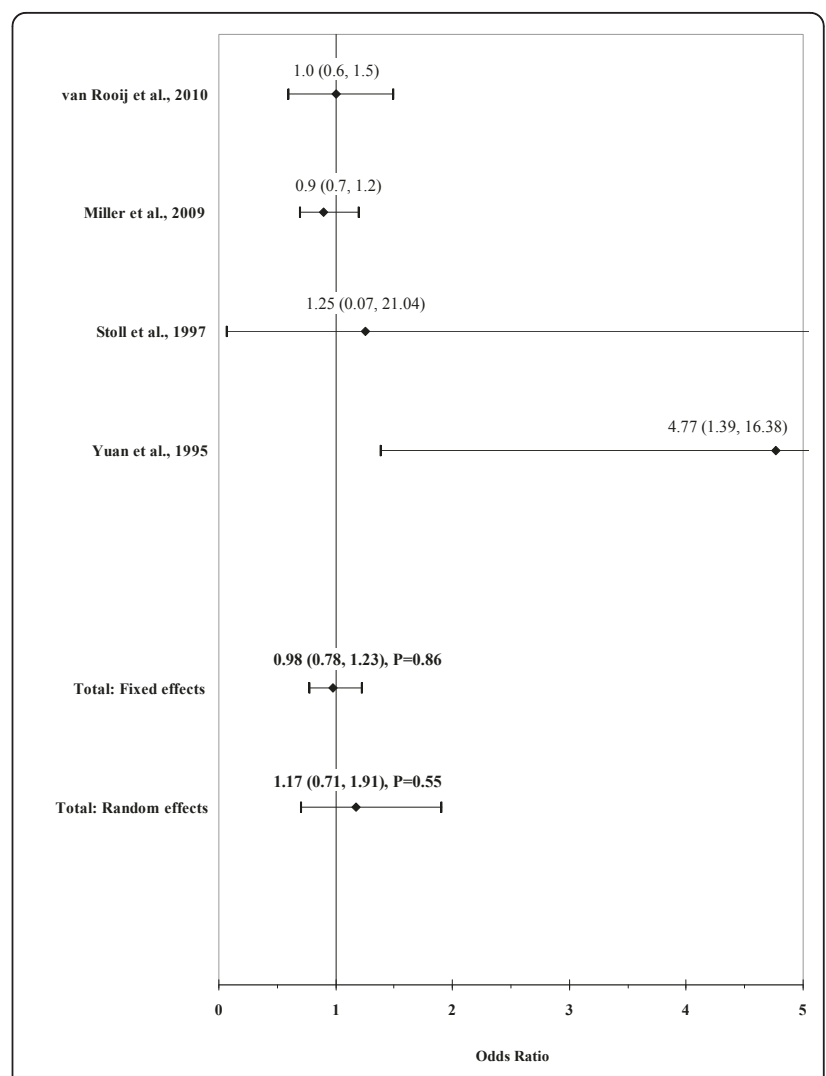

Figure 4 Forest plot for maternal alcohol consumption.

both groups $[7,30,34,37,38,40]$. Analyses, however, showed nearly the same results. Furthermore, two studies by Martínez-Frías et al. [44] and Sharpe et al. [45] were excluded because they grouped ARM with other congenital malformations (among others intestinal and tracheo-esophageal atresias) which might mix or dilute potential effects in case of diverse etiologies. The excluded studies did not find an association with the examined risk factors. In contrast, although Honein et al. [31] reported on the examination of "rectal atresia", a very rare subgroup of ARM, we included this study because it appears that the term of "rectal atresia" was used synonymously for ARM, given that the reported sample size, collected within one year, appear too high for rectal atresia and Honein et al. [31] compared their results with other studies reported on ARM. In general, there is no unique terminology for ARM that is used in the literature. Besides anorectal malformation, terms of anal atresia, anorectal atresia and imperforate anus can be found for this anomaly. Even ARM itself is a mixed group with isolated and associated malformations ranging from lower to higher forms with different genetic background [46].

Looking at some other gastro-intestinal malformations, maternal diabetes also seems to be a risk factor for 


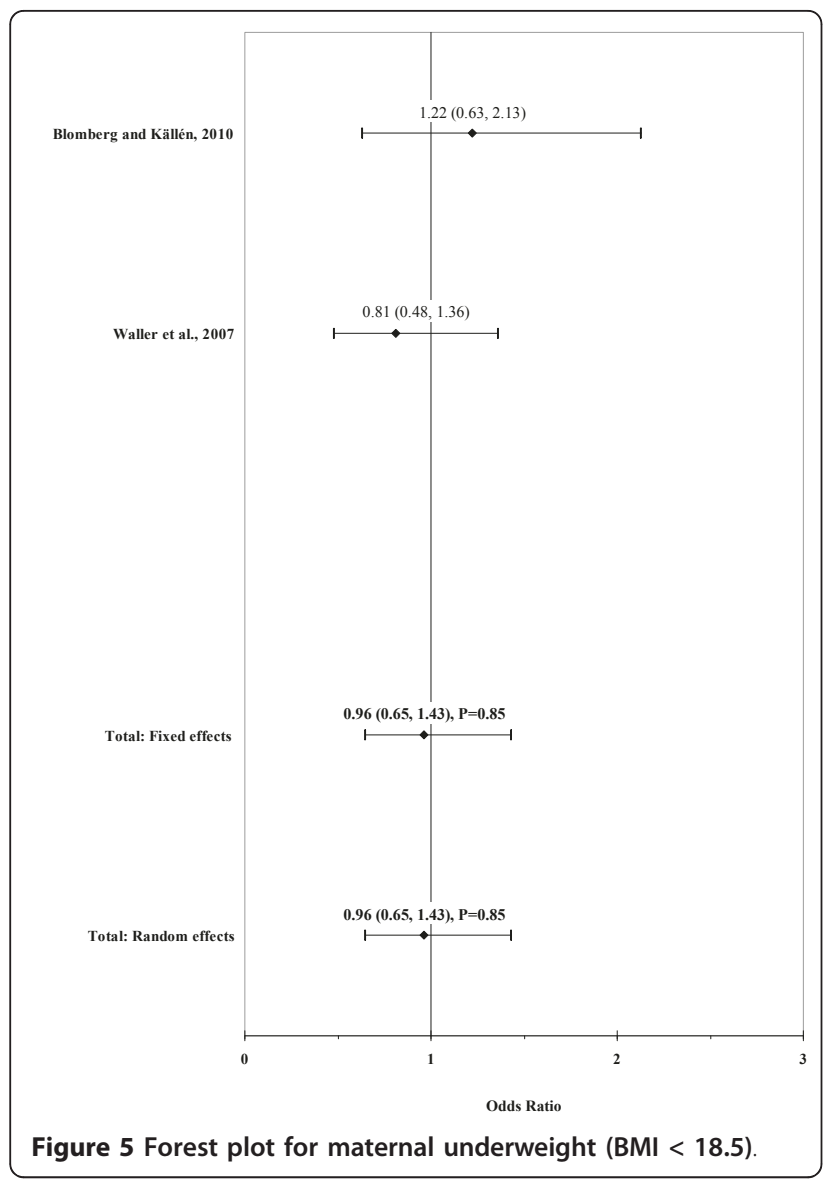

esophageal atresia $[25,32,34]$. However, no clear association was found with gastroschisis, omphalocele, smallintestinal atresia and duodenal atresia $[32,34,36,43]$. There is a suggestive association between maternal overweight and omphalocele [40], but not for the other defects. Maternal obesity also seems to be a risk factor for omphalocele, whereas an inverse association was found for gastroschisis $[47,48]$. The use of illicit drugs including cocaine, methamphetamines and marijuana during pregnancy was found to be associated with increased risk of gastroschisis [47] and the use of cocaine, amphetamines, decongestants and pseudoephedrine was associated with intestinal atresia [49-51]. Consistently increased risks for maternal smoking were only observed for pyloric stenosis [52]. For omphalocele and gastroschisis, no consistently increased risks were found regarding alcohol consumption [47].

The significant associations with ARM and some other gastro-intestinal malformations show that the rise in maternal overweight and obesity, as well as diabetes during the last decades are of relevance for these birth defects. For example, the prevalence of overweight (obesity) in adult females from the Netherlands increased from $30 \%(6 \%)$ in 1981 to $42 \%(12 \%)$ in 2004 [53]. In

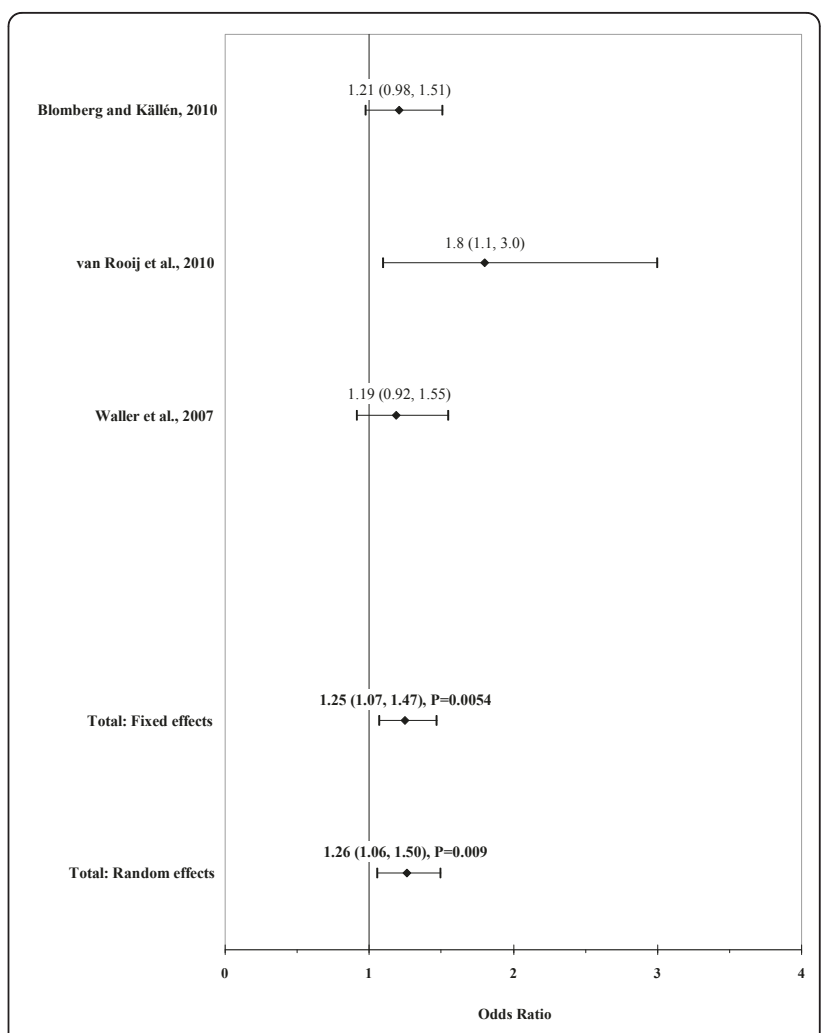

Figure 6 Forest plot for maternal overweight (BMI 25-29.9).

girls, overweight (obesity) prevalence doubled or even tripled from 1980 to 1997 and again from 1997 to 2002-2004. The same trend was observed in the United States where $17.9 \%$ of all school-age girls were overweighed (11.7\% obese) in $1999-2000$ and $22.3 \%$ were overweighed (13.6\% obese) in 2003-2004 [54]. During the same time, the number of women with diabetes was also increasing. The worldwide prevalence of type 1 and type 2 diabetes in women has doubled since the $1980 \mathrm{~s}$ $[55,56]$. The overall prevalence was estimated to be $2.2 \%$ worldwide in 1995 and is expected to be $2.8 \%$ in 2025 with a higher proportion in the developed countries than in the developing countries $(3.6 \%$ vs. $1.7 \%$ in $1995 ; 4.5 \%$ vs. $2.5 \%$ in 2025 ) [57]. Previous studies underline the need of substantial efforts to limit the obesity epidemic, which is also the main cause of the growing prevalence of diabetes among women in childbearing age $[58,59]$. Besides many other beneficial health effects, such efforts could substantially reduce the risk of ARM and other birth defects in the offspring. Although associations of maternal smoking and alcohol consumption with ARM do not seem to be established based on existing evidence, the adverse health effects of these habits on the embryonic development underline the importance of avoiding them throughout pregnancies and beyond. 


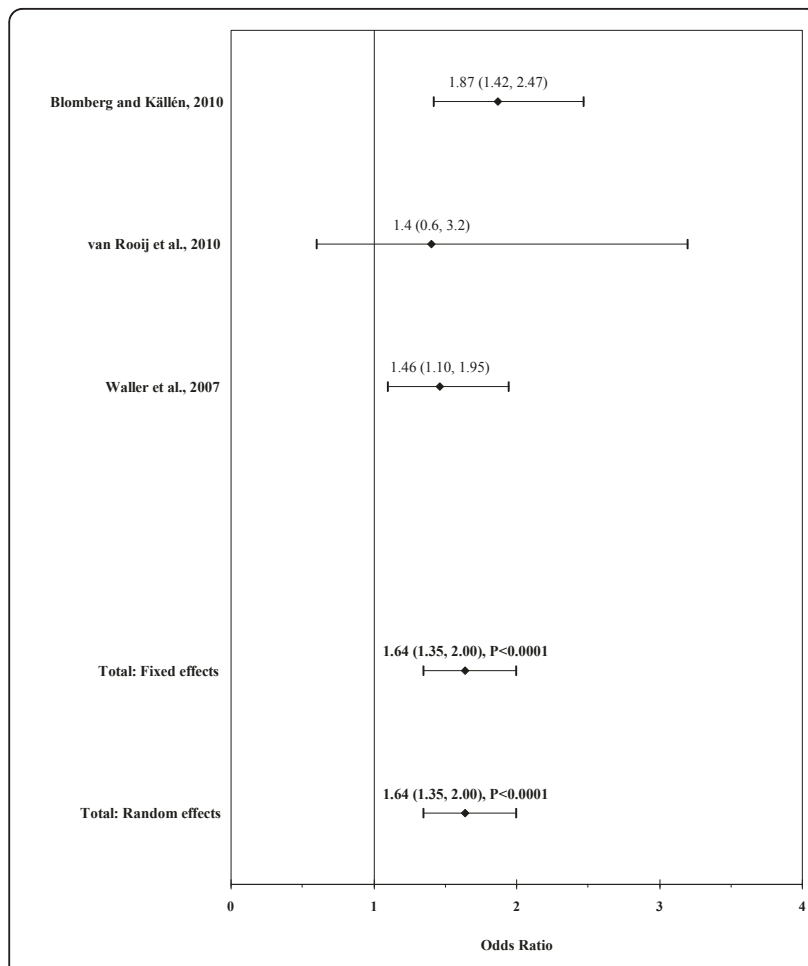

Figure 7 Forest plot for maternal obesity (BMI $\geq 30$ ).

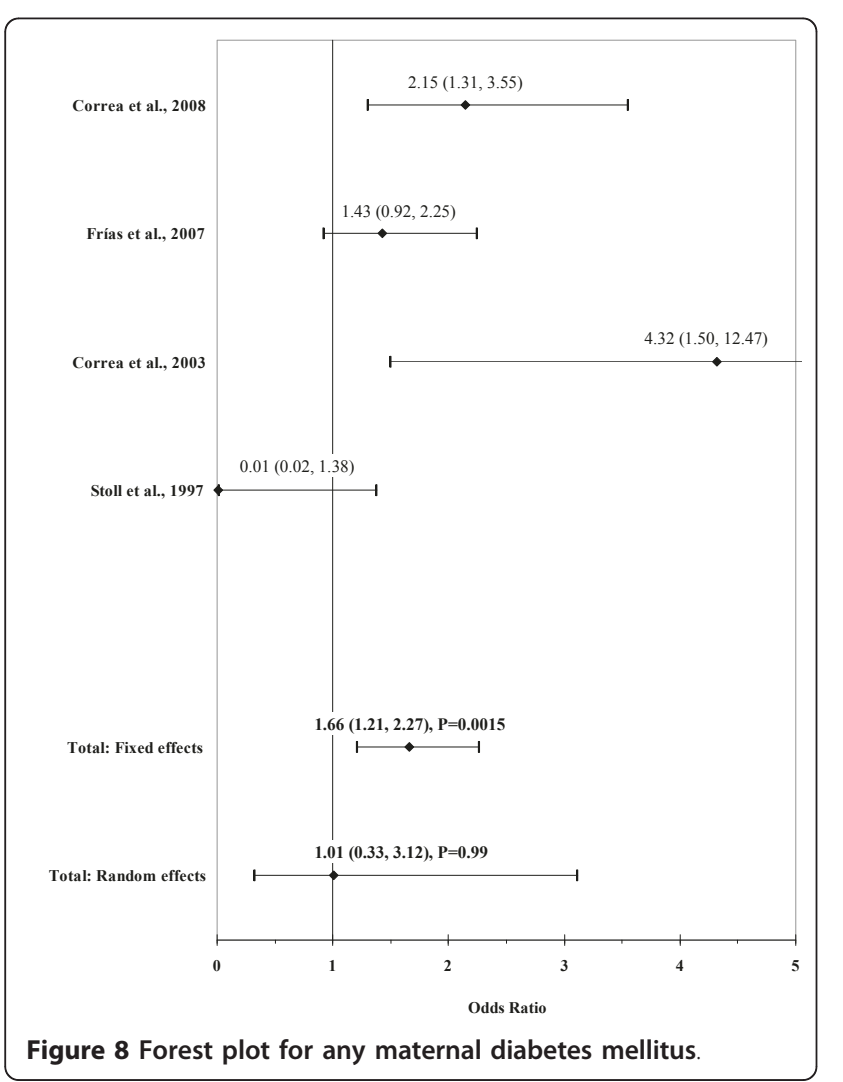

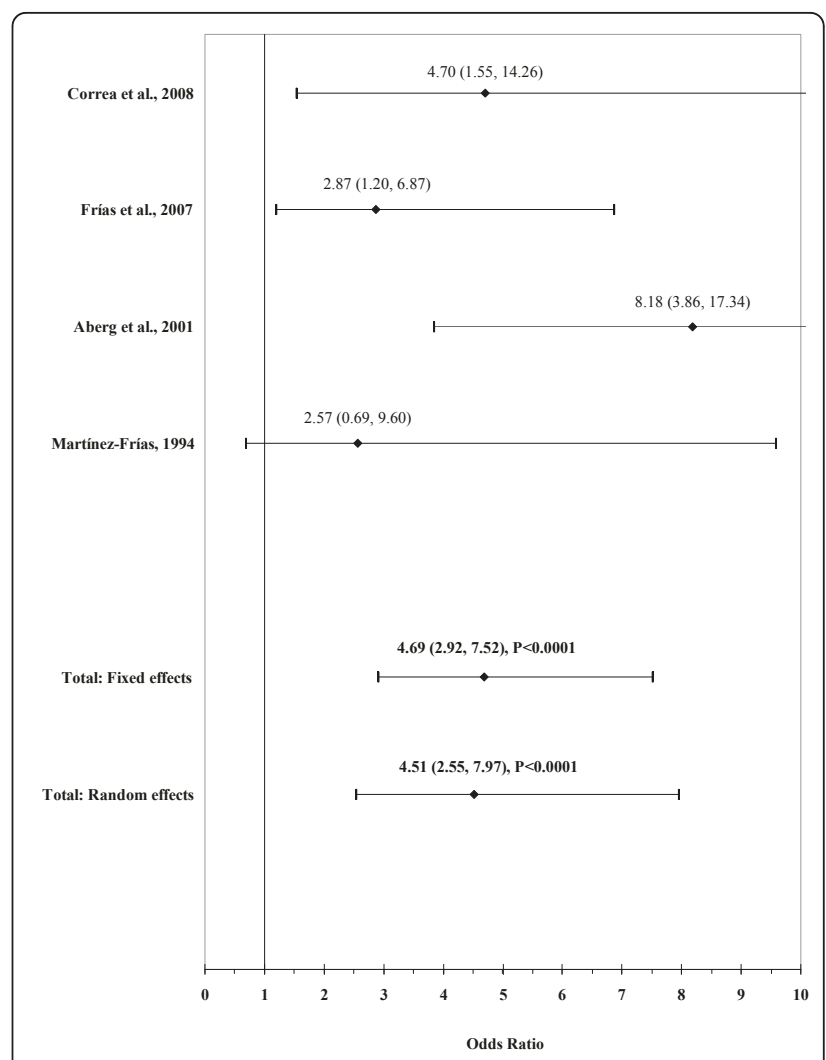

Figure 9 Forest plot for maternal pre-gestational diabetes.

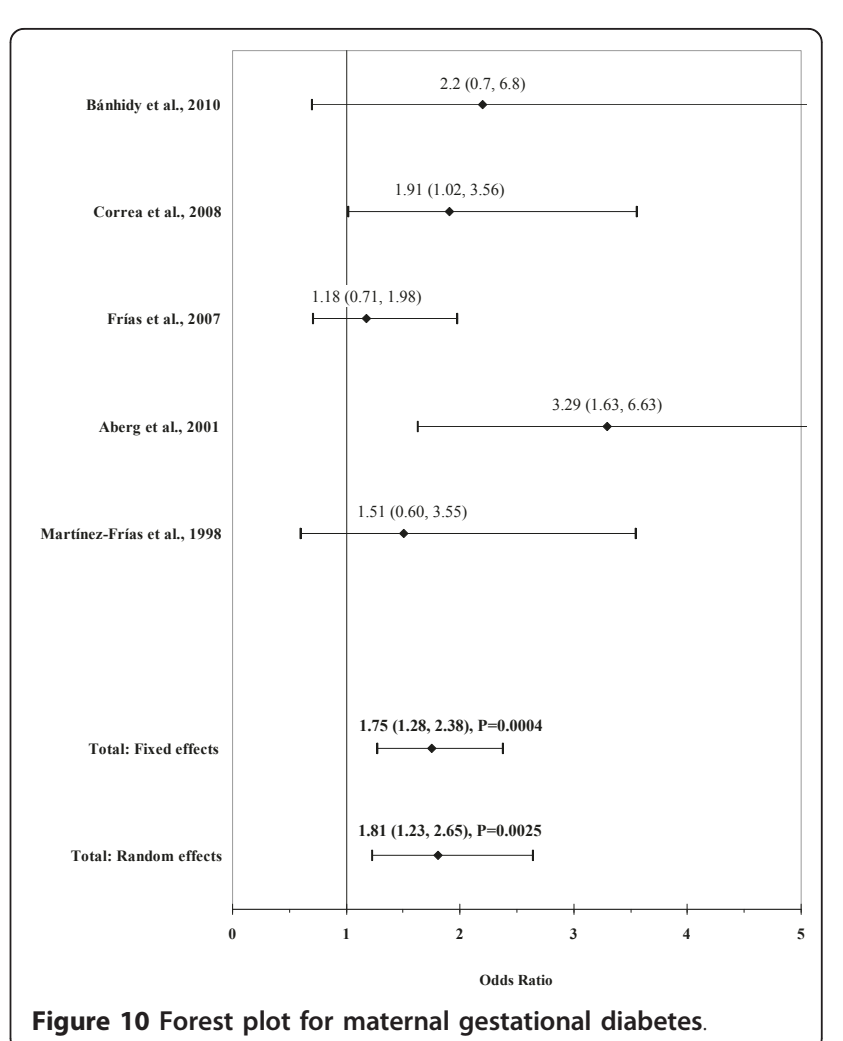


Our review has a number of limitations mostly resulting from the overall scarcity of published evidence. First, our meta-analysis was limited by the data provided in the individual studies. Not all studies provided risk estimates adjusted for potentially influential confounders, such as maternal age, ethnicity, education, parity, periconceptional smoking and folic acid intake [40] or maternal age, race/ethnicity, education, smoking, binge drinking, pregnancy BMI and periconceptional folic acid use [41]. Analyses of diabetes were only adjusted for BMI in the study by Correa et al. [34], known as potential influential confounder. Due to the small number of studies, we decided to pool adjusted and crude values for meta-analyses. Second, some studies used affected (malformed) control groups. Other studies used mixed controls of live-born malformed and healthy babies. A potential advantage of using malformed controls is potential reduction of response bias or recall bias that may occur when a non-malformed control group is used. On the other hand, observed associations may be biased if the risk factors of interest are also associated with the malformations of controls. Third, most sample sizes were small, so the power to detect associations was low. Fourth, despite the lack of indication of major publication bias, it is impossible to be ruled out completely, especially in the light of the low number of studies. Finally, although we searched in four databases (PubMed, EMBASE, ISI Web of Knowledge and the Cochrane library) and completed our search by reviewing related and cross-referencing literature, existence of relevant missing studies cannot be excluded.

To our knowledge, our article is the first systematic review and meta-analysis that provides an overview of the few available studies that reported on the association between prenatal environmental risk factors of the parents and ARM. Adequate evidence is still very limited. Therefore, further multicenter or register-based studies are needed to clarify the role of key risk factors for the development of ARM. One example is the recent establishment of the German Network for Congenital UroREctal Malformations (CURE-Net). The aim of this consortium is to collect data of affected newborns as well as of older patients with an anorectal malformation (ARM) or an exstrophy-epispadias complex (EEC) that allow to investigate molecular causes, clinical implications and psychosocial outcomes. For a standardized description of diagnostic subgroups, international classifications are used (Krickenbeck for ARM [60] and Gearhart \& Jeffs for EEC [61]). Associated malformations are collected with means of the London Dysmorphology Database [62] and environmental risk factors according to the core dataset of surveillance of congenital anomalies in Europe (EUROCAT) [63]. Nationwide data acquisition should enable to achieve a sample size that is large enough to clarify the role of key risk factors for the development for ARM and EEC in general but also for each subgroup separately. Furthermore, activities are ongoing aiming to expand such a register on an international level. The recently established International Consortium on Anorectal Malformations (ARM-Net), a European collaboration between France, Italy, Germany and the Netherlands, aims to identify genetic and environmental risk factors by data sharing and combined research activities [64]. Both consortia offer the unique opportunity to establish a basis for future research to overcome current scarcity of evidence in the field of ARM, especially from populations outside the United States.

\section{Acknowledgements}

$\mathrm{NZ}$ and EJ are members of the "Network for Systematic Investigation of the Molecular Causes, Clinical Implications and Psychosocial Outcome of Congenital Uro-Rectal Malformations (CURE-Net)" supported by a research grant (01GM08107) from the German Federal Ministry of Education and Research (Bundesministerium für Bildung und Forschung, BMBF): http:// www.cure-net.de.

\section{Authors' contributions}

Conception and design was done by HB. Literature review, data extraction and statistical analysis were carried out by NZ and EJ. Drafting of the article was done by NZ. Revision of the article was done by NZ, EJ and HB. All authors read and approved the final manuscript.

\section{Competing interests}

The authors declare that they have no competing interests.

Received: 24 January 2011 Accepted: 17 May 2011

Published: 17 May 2011

\section{References}

1. Forrester MB, Merz RD: Risk of Selected Birth Defects with Prenatal Illicit Drug Use, Hawaii, 1986-2002. J Toxicol Environ Health A 2007, 70:7-18.

2. International Clearinghouse Birth Defects Surveillance and Research. [http://www.icbdsr.org/].

3. Jenetzky E: Prevalence estimation of anorectal malformations using German diagnosis related groups system. Pediatr Surg Int 2007, 23:1161-5.

4. Cho S, Moore SP, Fangman T: One Hundred Three Consecutive Patients With Anorectal Malformations and Their Associated Anomalies. Arch Pediatr Adolesc Med 2001, 155:587-91.

5. Cuschieri A, EUROCAT Working Group: Descriptive Epidemiology of Isolated Anal Anomalies: A Survey of 4.6 Million Birth in Europe. Am J Med Genet 2001, 103:207-15.

6. Cuschieri A, EUROCAT Working Group: Anorectal anomalies associated with or as part of other anomalies. Am J Med Genet 2002, 110:122-30.

7. Correa A, Botto L, Liu Y, Mulinare J, Erickson JD: Do multivitamin supplements attenuate the risk for diabetes-associated birth defects? Pediatrics 2003, 111:1146-51.

8. Leck I, Lancashire RJ: Birth prevalence of malformations in members of different ethnic groups and in the offspring of matings between them, in Birmingham, England. J Epidemiol Community Health 1995, 49:171-9.

9. Louw JH: Congenital abnormalities of the rectum and anus. Curr Probl Surg 1965, 2:1-64.

10. Moore SW, Alexander A, Sidler D, Alves J, Hadley GP, Numanoglu A, Banieghbal B, Chitnis M, Birabwa-Male D, Mbuwayesango B, Hesse A, Lakhoo K: The spectrum of anorectal malformations in Africa. Pediatr Surg Int 2008, 24:677-83.

11. Murphy F, Puri P, Hutson JM, Holschneider AM: Incidence and frequency of different types and classification of Anorectal malformations. In Anorectal Malformations in Children: Embryology, Diagnosis, Surgical 
Treatment, Follow-up.. 1 edition. Edited by: Holschneider AM, Hutson JM. Berlin, Heidelberg, New York: Springer; 2006:163-184

12. Stephens FD, Smith ED, Paul NW: Anorectal malformations in children: Update 1988. Birth Defects Orig Artic Ser 1988, 24:1-604.

13. Fitzgerald MJT, Fitzgerald M: Human Embryology: A Human Approach. Philadelphia: Bailliere Tindall; 1994.

14. Froster UG, Wallner SJ, Reusche E, Schwinger E, Rehder H: VACTERL with hydrocephalus and branchial arch defects: prenatal, clinical, and autopsy findings in two brothers. Am J Med Genet 1996, 62:169-72.

15. Hutson JM, $\vee$ Putte SCJ, Penington E, Kluth D, Fiegel H: The Embryology of Anorectal Malformations. In Anorectal Malformations in Children: Embryology, Diagnosis, Surgical Treatment, Follow-up.. 1 edition. Edited by: Holschneider AM, Hutson JM. Berlin, Heidelberg, New York: Springer; 2006:49-63.

16. Kluth $D$, Hillen $M$, Lambrecht $W$ : The principles of normal and abnormal hindgut development. J Pediatr Surg 1995, 30:1143-7.

17. Martínez-Frías ML, Frías JL, Opitz JM: Errors of morphogenesis and developmental field theory. Am J Med Genet 1998, 76:291-6.

18. Nievelstein RAJ, Van der Werff JFA, Verbeek FJ, Valk J, Vermeij-Keers C: Normal and Abnormal Embryonic Development of the Anorektum in Human Embryos. Teratology 1998, 57:70-78.

19. Higgens JP, Simon GT: Quantifying heterogeneity in a meta-analysis. Stat Med 2002, 21:1539-58

20. Higgins JP, Thompson SG, Deeks JJ, Altman DG: Measuring inconsistency in meta-analyses. BMJ 2003, 327:557-60.

21. Ioannidis JP, Patsopoulos NA, Evangelou E: Uncertainty in heterogeneity estimated in meta- analyses. BMJ 2007, 335:914-6.

22. Begg CB, Mazumdar M: Operating characteristics of a rank correlation test for publication bias. Biometrics 1994, 50:1088-1101.

23. Egger M, Davey Smith G, Schneider M, Minder C: Bias in meta-analysis detected by a simple, graphical test. BMJ 1997, 315:629-634.

24. Aberg A, Westbom L, Källén B: Congenital malformations among infants whose mothers had gestational diabetes or preexisting diabetes. Early Hum Dev 2001, 61:85-95.

25. Bánhidy F, Acs N, Puhó EH, Czeizel AE: Congenital abnormalities in the offspring of pregnant women with type 1, type 2 and gestational diabetes mellitus: a population-based case-control study. Congenit Anom (Kyoto) 2010, 50:115-21.

26. Blomberg Ml, Källén B: Maternal Obesity and Morbid Obesity: the Risk for Birth Defects in the Offspring. Birth Defects Res A Clin Mol Teratol 2010, 88:35-40.

27. Cornel MC, Erickson JD, Khoury MJ, James LM, Liu Y: Population-based birth-defect and risk factor surveillance: data from the Northern Netherlands. Int J Risk Safety Med 1996, 8:197-209.

28. Matte TD, Mulinare J, Erickson JD: Case-Control Study of Congenital Defects and Parental Employment in Health Care. Am J Ind Med 1993, 24:11-23.

29. Schnitzer PG, Olshan AF, Erickson JD: Paternal occupation and risk of birth defects in offspring. Epidemiology 1995, 6:577-83.

30. Yuan P, Okazaki I, Kuroki Y: Anal atresia: effect of smoking and drinking habits during pregnancy. Jpn J Hum Genet 1995, 40:327-32.

31. Honein MA, Paulozzi LJ, Watkins ML: Maternal smoking and birth defects: validity of birth certificate data for effect estimation. Public Health Rep 2001, 116:327-35.

32. Frías JL, Frías JP, Frías PA, Martínez-Frías ML: Infrequently Studied Congenital Anomalies as Clues to the Diagnosis of Maternal Diabetes Mellitus. Am J Med Genet A 2007, 143A:2904-9.

33. Shiono PH, Klebanoff MA, Berendes HW: Congenital Malformations and Maternal Smoking During Pregnancy. Teratology 1986, 34:65-71.

34. Correa A, Gilboa SM, Besser LM, Botto LD, Moore CA, Hobbs CA, Cleves MA, Riehle-Colarusso TJ, Waller DK, Reece EA: Diabetes mellitus and birth defects. Am J Obstet Gynecol 2008, 199(237):e1-237-e9.

35. Herdt-Losavio ML, Lin S, Chapman BR, Hooiveld M, Olshan A, Liu X, DePersis RD, Zhu J, Druschel CM: Maternal occupation and the risk of birth defects: an overview from the National Birth Defects Prevention Study. Occup Environ Med 2010, 67:58-66.

36. Martínez-Frías ML, Bermejo E, Rodríguez-Pinilla E, Prieto L, Frías IL: Epidemiological Analysis of Outcomes of Pregnancy in Gestational Diabetic Mothers. Am J Med Genet 1998, 78:140-5.

37. Miller EA, Manning SE, Rasmussen SA, Reefhuis J, Honein MA: Maternal exposure to tobacco smoke, alcohol and caffeine, and risk of anorectal atresia: National Birth Defects Prevention Study 1997-2003. Paediatr Perinat Epidemiol 2009, 23:9-17.

38. Stoll C, Alembik Y, Roth MP, Dott B: Risk factors in congenital anal atresias. Ann Genet 1997, 40:197-204.

39. van Rooij IA, Wijers CH, Rieu PN, Hendriks HS, Brouwers MM, Knoers NV, de Blaauw I, Roeleveld N: Maternal and Paternal Risk Factors for Anorectal Malformations: A Dutch Case-Control Study. Birth Defects Res A Clin Mol Teratol 2010, 88:152-8.

40. Waller DK, Shaw GM, Rasmussen SA, Hobbs CA, Canfield MA, Siega-Riz AM, Gallaway MS, Correa A, National Birth Defects Prevention Study: Prepregnancy Obesity as a Risk Factor for Structural Birth Defects. Arch Pediatr Adolesc Med 2007, 161:745-50.

41. van Gelder MM, Reefhuis J, Caton AR, Werler MM, Druschel CM, Roeleveld N, National Birth Defects Prevention Study: Maternal Periconceptional Illicit Drug Use and the Risk of Congenital Malformations. Epidemiology 2009, 20:60-6.

42. Angerpointer T, Radtke W, Murken JD: Catamnestic investigations in children with malformations of the gastrointestinal tract and the abdominal wall. Z Kinderchir 1981, 32:129-44.

43. Martínez-Frías ML: Epidemiological Analysis of Outcomes of Pregnancy in Diabetic Mothers: Identification of the Most Characteristic and Most Frequent Congenital Anomalies. Am J Med Genet 1994, 51:108-13.

44. Martínez-Frías ML, Bermejo E, Rodríguez-Pinilla E, Frías JL: Risk for Congenital Anomalies Associated with Different Sporadic and Daily Doses of Alcohol Consumption during Pregnancy: A Case-Control Study. Birth Defects Res A Clin Teratol 2004, 70:194-200.

45. Sharpe PB, Chan A, Haan EA, Hiller JE: Maternal Diabetes and Congenital Anomalies in South Australia 1986-2000: A Population-Based Cohort Study. Birth Defects Res A Clin Mol Teratol 2005, 73:605-11.

46. Falcone RA Jr, Levitt MA, Peña A, Mates M: Increased heritability of certain types of anorectal malformations. J Pediatr Surg 2007, 42:124-8.

47. Frolov $P$, Alali J, Klein MD: Clinical risk factors for gastroschisis and omphalocele in humans: a review of the literature. Pediatr Surg Int 2010, 26:1135-48.

48. Stothard K, Tennant PW, Bell R, Rankin J: Maternal overweight and obesity and the risk of congenital anomalies: a systematic review and meta-analysis. JAMA 2009, 301:636-50.

49. Hoyme HE, Jones KL, Dixon SD, Jewett T, Hanson JW, Robinson LK, Msall ME, Allanson JE: Prenatal cocaine exposure and fetal vascular disruption. Pediatrics 1990, 85:743-7.

50. Werler MM, Sheehan JE, Mitchell AA: Association of vasoconstrictive exposures with risks of gastroschisis and small intestinal atresia. Epidemiology 2003, 14:349-54.

51. Werler MM, Sheehan JE, Mitchell AA: Maternal medication use and risks of gastroschisis and small intestinal atresia. Am J Epidemiol 2002, 155:26-31.

52. Sørensen HT, Nørgård B, Pedersen L, Larsen H, Johnsen SP: Maternal smoking and risk of hypertrophic infantile pyloric stenosis: 10 year population based cohort study. BMJ 2002, 325:1011-2.

53. Schokker DF, Visscher TL, Nooyens AC, van Baak MA, Seidell JC: Prevalence of overweight and obesity in the Netherlands. Obes Rev 2006, 8:101-8.

54. Lobstein T, Jackson-Leach R: Child overweight and obesity in the USA: prevalence rates according to IOTF definitions. Int Pediatr Obes 2007, 2:62-4.

55. Lusignan S, Sismanidis C, Carey IM, DeWilde S, Richards N, Cook DG: Trends in the prevalence and management of diagnosed type 2 diabetes 19942001 in England and Wales. BMC Fam Pract 2005, 6:13.

56. Patterson CC, Dahlquist GG, Gyürüs E, Green A, Soltész G, EURODIAB Study Group: Incidence trends for childhood type 1 diabetes in Europe during 1989-2003 and predicted new cases 2005-20: a multicentre prospective registration study. Lancet 2009, 373:2027-33.

57. King H, Aubert RE, Herman WH: Global burden of diabetes, 1995-2025: prevalence, numerical estimates, and projections. Diabetes Care 1998, 21:1414-31.

58. Deckelbaum RJ, Williams CL: Childhood obesity: the health issue. Obes Res 2001, 9(Suppl 4):239-243.

59. Kramer H, Cao G, Dugas L, Luke A, Cooper R, Durazo-Arvizu R: Increasing $\mathrm{BMI}$ and waist circumference and prevalence of obesity among adults with Type 2 diabetes: the National Health and Nutrition Examination Surveys. J Diabetes Complications 2010, 24:368-74.

60. Holschneider A, Hutson J, Peña A, Beket E, Chatterjee S, Coran A, Davies M, Georgeson K, Grosfeld J, Gupta D, Iwai N, Kluth D, Martucciello G, Moore S, 
Rintala R, Smith ED, Sripathi DV, Stephens D, Sen S, Ure B, Grasshoff S, Boemers T, Murphy F, Söylet Y, Dübbers M, Kunst M: Preliminary report on the International Conference for the Development of Standards for the Treatment of Anorectal Malformations. J Pediatr Surg 2005, 40:1521-6.

61. Gearhart JP: The bladder exstrophy-epispadias-cloacal exstrophy complex. In Pediatric Urology. Volume Chapter 32. Edited by: Gearhart JP, Rink RC, Mouriquand PDE. Philadelphia: W.B. Saunders Co; 2001:511-546.

62. Winter RM, Baraitser M, Douglas JM: A computerised data base for the diagnosis of rare dysmorphic syndromes. J Med Genet 1984, 21:121-3.

63. EUROCAT - European surveillance of congenital anomalies. [http://www. eurocat-network.eu/]

64. Wijers $\mathrm{CH}$, de Blaauw I, Marcelis CL, Wijnen RM, Brunner H, Midrio P, Gamba P, Clementi M, Jenetzky E, Zwink N, Reutter H, Bartels E, GrasshoffDerr S, Holland-Cunz S, Hosie S, Märzheuser S, Schmiedeke E, Crétolle C, Sarnacki S, Levitt MA, Knoers NV, Roeleveld N, van Rooij IA: Research perspectives in the etiology of congenital anorectal malformations using data of the International Consortium on Anorectal Malformations: evidence for risk factors across different populations. Pediatr Surg Int 2010, 26:1093-9.

doi:10.1186/1750-1172-6-25

Cite this article as: Zwink et al:: Parental risk factors and anorectal malformations: systematic review and meta-analysis. Orphanet Journal of Rare Diseases 2011 6:25

\section{Submit your next manuscript to BioMed Central} and take full advantage of:

- Convenient online submission

- Thorough peer review

- No space constraints or color figure charges

- Immediate publication on acceptance

- Inclusion in PubMed, CAS, Scopus and Google Scholar

- Research which is freely available for redistribution

Submit your manuscript at www.biomedcentral.com/submit 